TITLE:

\title{
Region-based automatic mapping of tsunami-damaged buildings using multi-temporal aerial images
}

$\operatorname{AUTHOR}(\mathrm{S})$ :

Susaki, Junichi

CITATION:

Susaki, Junichi. Region-based automatic mapping of tsunamidamaged buildings using multi-temporal aerial images. Natural Hazards 2015, 76(1): 397-420

ISSUE DATE:

2015-03

URL:

http://hdl.handle.net/2433/199827

\section{RIGHT:}

The final publication is available at Springer via http://dx.doi.org/10.1007/s11069-0141498-4.; この論文は出版社版でありません。引用の際には出版社版をご確認ご利用くだ さい。; This is not the published version. Please cite only the published version. 


\section{Region-based Automatic Mapping of Tsunami-damaged Buildings Using Multi-temporal Aerial Images}

3

\author{
Junichi Susaki \\ Department of Civil and Earth Resources Engineering, \\ Graduate School of Engineering, Kyoto University \\ Kyotodaigaku Katsura, Nishikyo-ku, Kyoto 615-8540, Japan; \\ E-Mail: susaki.junichi.3r@kyoto-u.ac.jp; Tel.\& Fax: +81-75-383-3300
}

Abstract: After a disaster, prompt distribution of information is critical for national or local governments to plan the disaster response and recovery measures. In the case of a tsunami, information about buildings destroyed by the waves is required. Here we present a method that identifies individual damaged buildings by using aerial images obtained pre- and post-tsunami. The method utilizes significant height changes in building regions to assess the damage. Stereo aerial images are used to generate a digital surface model (DSM) of the area. We assume two cases: if GIS (geographic information system) data (building region data) are available, we use them; if GIS data are unavailable, we instead use segmented results and a filtered DSM. In each case regions corresponding to buildings are identified in the pre-tsunami image. Damaged regions are then extracted by considering the height change within a building region between the pre- and post-disaster images. Horizontal shifts resulting from land deformation caused by the earthquake are automatically estimated by an existing algorithm such as scale-invariant feature transform (Lowe, D., 2004. International Journal of Computer Vision, 60(2), 91-110). Validation showed that the proposed method extracted damaged buildings with high accuracy ( $94 \%$ to $96 \%$ in number; $96 \%$ to $98 \%$ in area) when GIS data are available and with lower accuracy (69\% to $79 \%$ in area) when GIS data are unavailable. In addition, we found that horizontal shifts between pre- and post-disaster should be considered to extract the damaged buildings. We conclude that our method can automatically generate effective maps of buildings damaged not only by tsunamis but also by other disasters.

Keywords: remote sensing;mapping; extraction; change detection; damaged building; aerial image.

\section{Introduction}

Following the Great East Japan Earthquake of March 11, 2011, it has been estimated there is a likelihood of approximately $60 \%$ to $70 \%$ that an earthquake with a magnitude of 8 to 9 will occur in the Nankai Trough within 30 years (Headquarters for Earthquake Research Promotion, 2013). A technique for rapidly mapping damaged areas and buildings is required to reduce the confusion after such disasters. Damage mapping supports local government decisions about evacuation and recovery. Following the Great East Japan Earthquake, many satellite and airborne images were analyzed in order to understand which areas were damaged by the resulting tsunami. The traditional approach for damage mapping is to manually delineate the damaged buildings or districts using remotely sensed images. Although this technique is still useful, it is extremely time consuming, especially for a large area. 
To improve the efficiency of damage mapping after disasters, various approaches for automatically detecting damaged buildings or districts have been investigated. Studies have used (1) optical images for pre- and postdisaster (Tsusui et al., 2007), (2) synthetic aperture radar (SAR) images for pre-and post-disaster (Dekker, 2011; Chen and Sato, 2013; Sato, 2012; Yamaguchi, 2012; Watanabe et al. 2012; Liu and Yamazaki, 2011; Liu et al., 2012; Chini et al., 2012), (3) optical images for pre-disaster and SAR images for post-disaster (Wang and Jin, 2012), (4) optical images and SAR images for post-disaster only (Chini et al., 2013), and (5) SAR images for postdisaster (Li et al., 2012).

Since the Hyogoken-Nanbu Earthquake in Japan in 1995, space-borne SAR has been widely used to analyze the damage by calculating the correlation between pre- and post-earthquake data (Yonezawa and Takeuchi, 2001) and by looking for intensity changes (Matsuoka and Yamazaki, 2004). Brunner has reported an approach that combines information from very high spatial resolution multispectral and SAR images. Backscattering from buildings obtained from SAR is compared with the results of simulations using parameters obtained from optical images (Brunner et al., 2010). Space-borne polarimetric SAR (PolSAR) has also shown potential for extracting damaged areas by using four scattering components calculated from the original fully polarimetric data (Chen and Sato, 2013; Sato, 2012; Yamaguchi, 2012; Watanabe et al. 2012) or polarization orientation angle (Chen and Sato, 2013) to highlight the differences between the scattering before and after the disaster.

In our work, we initially analyzed the pre- and post-disaster images used by Chen and Sato (2013), which were acquired by the Advanced Land Observing Satellite (ALOS) / Phased Array Type L-band SAR (PALSAR) sensor. We used Ishinomaki city, Miyagi prefecture, Japan, as one of the study areas. This area was severely damaged by the 2011 tsunami. We examined three sets of features: scattering intensity of the three components $\mathrm{HH}(\mathrm{H}-$ polarization receiving and H-polarization transmitting), VV (V-polarization receiving and V-polarization transmitting) and HV (H-polarization receiving and V-polarization transmitting) (double bounce scattering, surface scattering and volume scattering) analyzed by Yamaguchi et al. (2011) and variance of polarization orientation angle. However, none of these features derived from PolSAR data clearly delineates damaged areas when compared to pre- and post-disaster optical images. Thus, we turned our attention to multi-temporal optical images as a source of data for automatic damage mapping.

It is customary in Japan to report the damage due to a disaster, such as an earthquake, tsunami or landslide, by referring to the number, rather than the area, of the buildings damaged. From this viewpoint, optical images, especially aerial images, are capable of meeting such requirements because they have a much higher spatial resolution than SAR images, often as high as $15 \mathrm{~cm}$ per pixel. High spatial resolution optical images can provide not only two-dimensional (2D) information but also three-dimensional (3D) information through a digital surface model (DSM), generated by a stereo matching approach. Height information can be effectively used to extract damaged buildings. Tong et al. (2012) used differences between the 3D coordinates of building corners pre- and post- earthquake to identify eight damaged buildings. Tian et al. (2013) detected building and forest changes by examining brightness and height changes derived from DSMs, generated from satellite imagery. Airborne light detection and ranging (LiDAR) can measure elevation and detect inundated or deformed areas (Persi, et al., 2013), and segmented point clouds can be classified and used to identify building damage (Khoshelham, et al., 2013). However, the opportunities to obtain LiDAR data soon after the 2011 disaster were much fewer than the opportunities to obtain optical images, so we focus on the optical images as a 3D data source. 
The extraction of damaged buildings is more difficult than simple land cover change detection because the land surface may be deformed by disasters. Vertical and horizontal shifts may take place but we focus on horizontal shifts for simplicity. Even though each post-disaster image is co-registered to a pre-disaster image using ground control points (GCPs), a relative horizontal shift may remain because the shift is not constant throughout the study area. Accurate extraction of damaged buildings, therefore, depends on information about such horizontal shifts. High spatial resolution optical images can provide this horizontal shift information as long as tie points between two images registered to the world coordinate system (WGS 84) can be found.

In this paper, we propose a novel method for extracting individual buildings damaged by a disaster using stereo aerial images. Relative horizontal shifts between pre- and post-disaster configurations are automatically estimated by finding tie points between two images. Height changes, estimated by comparison of pre- and post-disaster DSMs generated from stereo aerial images, are used to assess the damage done to a building. In the identification of building regions we consider two cases: either geographic information system (GIS) data are available, or they are not. If building region data are available, we can estimate the number and area of damaged buildings. However, the generation of building region data is costly and thus we cannot expect that such data will be provided for all the coastal regions in Japan. Even when building region data are unavailable, object-based classification can identify regions corresponding to buildings (Tuia et al., 2009; Novack et al., 2011), and we have developed a building segmentation algorithm that is robust to shadow (Susaki, 2012a). Filtering of the DSM can contribute to the selection of building regions from among the segmented regions. When building region data are unavailable, we can estimate the area, but not the number, of damaged buildings.

The remainder of this paper is organized as follows. After the study area is introduced in Section 2, the proposed method and experimental results are reported in Section 3. They are discussed in Section 4, and finally, some conclusions are given in Section 5.

\section{Study Area and Data Used}

We selected two study areas severely damaged by the 2011 tsunami: Yuriage district, Natori city (Study Area 1) and Ishinomaki city (Study Area 2), Miyagi prefecture, Japan. Aerial stereo pre- and post-tsunami images of the study areas were available. Each study area has three data sets: pre-tsunami $\left(t_{1}\right)$, one day post-tsunami $\left(t_{2}\right)$, and approximately two months post-tsunami $\left(t_{3}\right)$. For Study Area $1 t_{1}, t_{2}$ and $t_{3}$ were May 15, 2009, March 12, 2011, and May 26, 2011, respectively. For Study Area $2 t_{1}, t_{2}$ and $t_{3}$ were May 18, 2009, March 12, 2011, and May 18, 2011, respectively. The images are shown in Figure 1.

\section{Method and Results}

\subsection{Outline}

In the present paper, we focus on the extraction of buildings damaged by disasters from the data observed from the sky or space. The technique is effective to extract the buildings that show a significant height change between pre- and post-disaster. It is true that some buildings damaged by disasters such as earthquakes and tsunamis have no or very little height change, but we define a damaged building as a building whose height 
change exceeds a designated value. We therefore exclude buildings damaged by disaster but whose heights are unchanged. The proposed method combines 2D data of a building area with height data from DSM generation to create a 3D model of objects. Horizontal shifts between pre- and post-disaster configurations are automatically estimated. We assume that the DSM is generated from stereo aerial images, and that the horizontal and vertical accuracies may not be high. Comparison of pre- and post-disaster heights of a specific pixel of images may falsely extract pixels as damaged areas because of the inaccurate DSM. Instead, by comparing pre- and postdisaster heights in regions, damaged buildings can be identified more robustly. When GIS data of building regions are available, they are used for 2D data. When they are unavailable, segmentation of a 2D image and filtering of the DSM are implemented. The segmented regions are masked using the results of DSM filtering, and they then are used for 2D data.

Figure 2 shows a flowchart of the proposed method. Our method has four main sub-processes: (1) automatic estimation of horizontal shifts; (2) automatic generation of DSMs from stereo aerial images acquired pre- and post-disaster; (3) identification of likely building areas through segmentation of the pre-disaster aerial images, filtering of the pre-disaster DSM and masking both results; and (4) comparison of the calculated heights in building regions pre- and post the disaster to identify likely areas of damage. When GIS data of building regions are available, Step 3 is skipped.

\subsection{DSM Generation}

Stereo aerial images can generate DSMs through photogrammetric processing. Tie points are used to determine the relative positions and orientations of cameras (relative orientation). After a point existing in more than one image is obtained, the world coordinates of the point can be restored by using external orientation parameters. Software that automatically obtains such points and generates DSMs is now available. In this research, we assume that the DSM is automatically generated from stereo aerial images by using existing software.

Match-AT 5.4 (Inpho, 2013a) and Match-T 5.4 (Inpho, 2013b) were used to generate DSMs. To automatically find tie points, it uses two matching algorithms: feature-based matching (FBM) and least squares matching (LSM). FBM does not need accurate initial approximations and finds rough matches quickly. After the rough matches have been found LSM, which needs accurate initial approximations, is implemented to find accurate matches using a $21 \times 21$ pixel window. The input data for the software are digital images and camera calibration reports.

Figure 3 shows DSMs of the study areas. Orthogonally projected images were generated by using a 50-m mesh digital elevation model (DEM) published by the Geospatial Information Authority of Japan (GIA of Japan, 2013c). The DEM was generated from 1:25,000 topographic maps. As a result, three grid-based DSMs at $t_{1}, t_{2}$ and $t_{3}$ were available. The resolution of the DSM generated in this research was $50 \mathrm{~cm}$. It was difficult to evaluate the accuracy of the generated DSMs because no reference data were available. However, in another study, we evaluated the DSMs generated by using the same software with airborne LiDAR data from another study area (Kyoto, Japan) by using 18 points where road width was not less than $5.0 \mathrm{~m}$. The standard deviation of the height change between the LiDAR data and the DSM was $0.71 \mathrm{~m}$ (Susaki et al., 2013). Note that because ground survey using GPS equipment was difficult at $t_{2}$, absolute orientation was not applied to the images taken at $t_{2}$.

\subsection{Horizontal Shift Detection}


We examined horizontal shifts caused both by the disaster and by the lack of absolute orientation. We manually took samples from pre- and post-tsunami images, and recorded the horizontal shift coordinates. Figures 4(a) and 4(b) show the horizontal shifts in Study Areas 1 and 2, respectively. The horizontal shift vectors between $t_{1}$ and $t_{3}$ were $(1.10 \mathrm{~m},-1.97 \mathrm{~m})$ and $(4.87 \mathrm{~m},-1.27 \mathrm{~m})$ for Study Areas 1 and 2, respectively. Both aerial images observed at $t_{1}$ and $t_{3}$ were registered through absolute orientation. The results agree well with the actual horizontal shifts obtained by GPS measurements at the nearest base stations to Study Areas 1 and 2, which were $3.08 \mathrm{~m}$ and $4.25 \mathrm{~m}$ in the east-southeast direction, respectively (GIA of Japan, 2013a; GIA of Japan, 2013b). On the other hand, the geolocational accuracies of aerial images taken at $t_{2}$ are not high because of the lack of absolute orientation. The horizontal shifts between $t_{1}$ and $t_{2}$ can be a mix of actual horizontal shifts and registration errors. Regardless of whether the absolute orientation is available, tie points between pre- and post-disaster images need to be found.

Automatic extraction of tie points has been very popular, and scale-invariant feature transform (SIFT), developed by (Lowe, 2004), is still one of the most useful algorithms for automatically extracting tie points. In the proposed method, SIFT is used to detect horizontal shifts between pre- and post-disaster images. The keypoints from the whole of the study area were sorted in ascending order of feature distance. Finally, keypoints were selected as long as newly selected keypoints were at least $5.0 \mathrm{~m}$ away from already selected keypoints. The maximum number of keypoints selected was 30. The relative shifts between keypoints were used as horizontal shifts caused by the earthquake. Because local horizontal shifts are fluctuating, the average should be used to represent the horizontal shift in the study area. The average horizontal shifts obtained by automatic and manual detections for Study Areas 1 and 2 are shown in Tables 1 and 2, respectively.

\subsection{Building Region Data}

Building region data are necessary to achieve highly accurate extraction of damaged buildings. Gamba et al. (2007) used GIS data for selecting training data sets and damage mapping. In addition, as mentioned in the introduction, damage to buildings caused by disasters is often reported by giving the number of damaged buildings. Without GIS data, it will be quite difficult to estimate this number. Therefore, GIS-based building region data should be provided with any rapid mapping system that is put into use. However, it is time-consuming to generate GIS data by delineating building boundaries on analogue maps and digitizing them. So we propose to generate building region data by mixing segmentation and filtering techniques

Many segmentation algorithms and software applications are now available. The ideal for disaster damage assessment would be that the segmentation units correspond to buildings. But in actuality, regions corresponding to buildings, roofs or parts of roofs may be segmented. It may, therefore, be necessary to develop better methods of segmentation. However, in this research, we do not develop a new segmentation method; instead we utilize existing segmentation algorithms or software. As a consequence, we assess disaster damage not in terms of the number of damaged buildings, but in terms of the area of damaged buildings, when building region data are generated using segmentation techniques.

In this research, we used two example methods for segmentation. In one method, we use ENVI EX (Version 4.8) (ENVI EX, 2009) which segments regions using a gradient map and watershed algorithm (Jin, 2009). The other method is an algorithm proposed by Susaki (2012a). The algorithm is designed to segment regions having a 
rough texture or that are overcast by shadow, both of which are difficult to segment using other algorithms and software. Quantization using several interval widths is applied during segmentation, and for each quantization, areas with homogeneous values are labeled in an image. Edges determined from the homogeneous areas obtained at each quantization are then merged, and frequently observed edges are extracted. By using a "rectangular index", regions whose shapes are close to being rectangular are selected as buildings. The same thresholds that were used by Susaki (2012a) were used in this research, except for the minimum rectangular index. This parameter has a range from 0 to 1 , where a higher index means the region's shape is closer to a rectangle. Susaki (2012a) used 0.45 , but it was set to 0.1 in this research to extract even regions whose shapes are far from rectangular, because such buildings were found in the study area. The "feature extraction" function in the ENVI EX software requires the setting of two parameters, "Scale Level" and "Merge Level”. Both have the range from 0.0 to 100.0, and are related to determine the number of segments. Higher values of the parameters generate fewer segments. From an empirical examination, these parameters were set to 50 and 80, respectively.

Segmentation results may include objects other than buildings. Filtering of the DSM can help in excluding the ground points from original point clouds. There are many filtering algorithms (Sithole and Vosselman, 2005; Mongus and Žalik, 2012; Susaki, 2012b) and in the proposed procedure, we use the filtering algorithm of Susaki (2012b). This slope-based algorithm utilizes planar surface features and connectivity with locally lowest points to improve the extraction of ground points. A slope parameter used in the proposed algorithm is updated after an initial estimation of the DSM, and thus local terrain information can be included. As a result, the proposed algorithm can extract ground points from areas where different degrees of slope variation are interspersed.

\subsection{Vegetation and Waterbody Exclusion}

Because extraction of damaged buildings is on the focus of the present research, vegetation and waterbodies should be excluded. Vegetation regions can be removed when examining regions to determine whether they are damaged buildings, as described in Subsection 3.5. It is widely reported that a combination of red and nearinfrared band reflectance is effective for extracting vegetation. Because aerial images usually have no nearinfrared band, such an approach cannot be used. However, the brightness of RGB bands may be used instead to extract vegetation. Vegetation regions were empirically excluded as follows: pixels having (1) a red DN higher than 100, (2) a blue DN higher than 100, and (3) a ratio of green to blue DNs higher than 1.0 were labeled as vegetation pixels. Regions that contained more than $50 \%$ vegetation pixels were labeled as vegetation regions and excluded from results.

On the other hand, automatic generation of a waterbody mask image is challenging because the brightness of the water is not homogeneous. Waterbodies can be manually delineated, and then they are masked after extracting damaged regions. Waterbodies were manually delineated, and then masked after extracting damaged regions. It took approximately 5 min to delineate one study area.

\subsection{Extraction of Damaged Regions}

The necessity for mapping damaged buildings soon after a disaster is quite high. However, ground surveys using GPS equipment are, in general, difficult soon after a disaster, and thus the accuracy of 3D coordinates in the 
DSM may not be high. Therefore, we have to develop a method that is robust to 3D coordinate errors. In order to find an effective indicator to differentiate damaged regions from undamaged regions, we examined the height changes between pre- and post-tsunami configurations by using DSMs. Figures 5(a) and 5(b) show the differences in heights of damaged and undamaged buildings in Study Area 2, respectively. Study Area 2 was selected for this purpose because samples of undamaged buildings in Study Area 1 that remained after the tsunami were quite limited. In order to reduce the effect of geolocational errors, we used the images at $t_{1}$ and $t_{3}$, to which absolute orientation was applied, and obtained the samples after adjusting the image at $t_{3}$ to take into account the horizontal shift.

All of the samples except "Dmg1" in Figure 5(a) show a clear trend in height change for damaged buildings. The heights of the samples post-tsunami were almost zero because the buildings were completely lost. On the other hand, samples in Figure 5(b) do not show such a clear trend. However, we have to pay attention to the samples "Dmg1" in Figure 5(a). The samples were from a large building with approximately $28 \mathrm{~m} \times 45 \mathrm{~m}$ of flat roof. The scattergram of the samples "Dmg1" shows that less than half of them do not follow the trend. This may be caused by errors in restoring 3D coordinates of points included in the area where the damaged building used to exist. Points in flat areas where no object exists may have inaccurate 3D coordinates because false tie points may be selected due to lack of texture.

Considering these findings, the proposed method takes a following approach. We set three thresholds: (1) a window size to locate the new position of the same pixel $\left(T_{1}\right)$, (2) height change $\left(T_{2}\right)$ and (3) minimum percentage of damaged pixels per region $\left(T_{3}\right)$. As mentioned in Subsection 3.2, horizontal shifts between pre- and postdisaster images are automatically obtained. To locate damaged buildings, our method examines each pixel in the pre-disaster image. Then all pixels in a window surrounding the pixel position in the post-disaster image are examined. The pixel position may differ from the position in the pre-disaster image due to the horizontal shifts. The method locates the pixel whose height is closest to the height of the pre-disaster pixel. Therefore, we set a threshold window size $\left(T_{1}\right)$ for the examination. After examining each pixel in the pre-disaster image, the method assesses the damaged regions. If the height change between this pre-disaster pixel and the post-disaster pixel was larger than a threshold value $\left(T_{2}\right)$, the pre-disaster pixel is labeled as potentially damaged. After pixel-based processing, the method examines each region. Regions where the ratio of labeled pixels to the total number of pixels was higher than another threshold value $\left(T_{3}\right)$ were extracted as being damaged.

We determined the optimal values of three parameters required in the proposed method. A traditional validation approach is that half of the reference data are used for the parameter selection, and the remaining half of the reference data are used for the assessment. However, if we take samples of damaged and undamaged buildings from Study Area 1, the number of undamaged buildings is very small. Study Area 2 is partly flat and partly hilly, and almost all damaged buildings were in the flat area. In this case also, the number of undamaged buildings in the flat area is small. Therefore, it is impossible to reserve enough undamaged buildings to assess the parameters obtained by using the samples. Therefore, we took the following approach: the parameters were given specific values and the results were compared with the reference data. The optimal parameters were determined based on the statistical indices.

Four indices were used for pixel-by-pixel validation: precision (TP/(TP + FP)), recall (TP/(TP + FN)), F-value 
FN and TN denote true positive, false positive, false negative and true negative, respectively. Overall accuracy can be a good statistical index. However, reference maps show that the ratios of areas of damaged building to the entire area were not high: 23.2\% for Study Area 1 and 13.0\% for Study Area 2. Therefore, we used F-value to determine the optimal combination of the three thresholds.

We tried various values for the three parameters as follows: 1 pixel $\times 1$ pixel, $3 \times 3,5 \times 5,7 \times 7$ and $9 \times 9$ for $T_{1} ; 1.0 \mathrm{~m}, 1.5 \mathrm{~m}, 2.0 \mathrm{~m}$ and $2.5 \mathrm{~m}$ for $T_{2}$; and $25 \%, 50 \%$ and $75 \%$ for $T_{3}$. We found that the combination of $2.0 \mathrm{~m}$ for $T_{2}$ and $50 \%$ for $T_{3}$ was the best for all of window sizes. With $2.0 \mathrm{~m}$ for $T_{2}$, and $50 \%$ for $T_{3}$, the optimal window size $\left(T_{1}\right)$ for determining number and area of damaged buildings when GIS data were available was 1 pixel $\times 1$ pixel $(0.5 \mathrm{~m} \times 0.5 \mathrm{~m}$ : no search). When GIS data were unavailable, the optimal size depended on the data set: 5 pixel $\times 5$ pixel $(2.5 \mathrm{~m} \times 2.5 \mathrm{~m})$ for comparison of the images at $t_{1}$ and $t_{2}$ in Study Area 1,7 pixel $\times 7$ pixel $(3.5 \mathrm{~m}$ $\times 3.5 \mathrm{~m})$ for comparison of the images at $t_{1}$ and $t_{3}$ in Study Area 1 , and 9 pixel $\times 9$ pixel $(4.5 \mathrm{~m} \times 4.5 \mathrm{~m})$ for comparisons of the images at $t_{1}$ and $t_{2}$ and at $t_{1}$ and $t_{3}$ in Study Area 2. Figure 6 shows the accuracies obtained by changing the values of $T_{1}$ for fixed values of $T_{2}$ and $T_{3}(2.0 \mathrm{~m}$ and $50 \%)$.

\subsection{Validation}

The performance for extracting damaged buildings was evaluated for various horizontal shift estimates: (1) average of automatically detected shifts, (2) manually detected local shifts, (3) average of manually detected shifts, and (4) no shift. As shown in Figures 1(b) and 1(c), some $200 \mathrm{~m} \times 200 \mathrm{~m}$ grids had no manually detected local shift because of the lack of surviving buildings. In this case, the average of manually detected shifts was assigned. The results for the extraction of damaged buildings using GIS data are shown in Figures 7 and 8 for Study Areas 1 and 2, respectively. Figures 7(b)(d)(f) and 8(b)(d)(f) show the validation results. Figures 7(a) and 8(a), Figures 7(c) and 8(c), and Figures 7(e) and 8(e) are results obtained using the average of automatically detected shifts, the manually detected local shifts, and no shift, respectively. They were generated using 1 pixel $\times 1$ pixel for $T_{1}$.

Figure 9 includes the results generated by masking the segmentation results (Susaki, 2012a) with the filtering results (Susaki, 2012b). From these results, damaged buildings were extracted without GIS data (Figures 10 and 11). Figures 10(a) and 11(a), Figures 10(c) and 11(c), and Figures 10(e) and 11(e) are results obtained using the average of automatically detected shifts, the manually detected local shift, and no shift, respectively. They were generated using 7 pixels $\times 7$ pixels for $T_{1}$.

The proposed method was compared with an existing method for extracting damaged buildings. The method proposed by Tian et al. (2013) was selected because the concept is similar to that of the proposed method: it uses two DSMs generated from stereo satellite imagery and compares the height change in segmented regions. However, it evaluates change detection by combining both the height change and the brightness change in regions, while the proposed method considers only the height change. In the experiment, the threshold for the change probability map was set to 2.0 by referring to the validation results of both study areas. For reference, Tian et al. (2013) used 0.5 for industrial areas and 0.9 for forest areas. The results obtained by using the method of Tian et al. (2013) are shown in Figure 12. Figures 13 and 14 show the effects of horizontal shifts. Figure 13 shows the accuracies obtained by using GIS data. Figure 14 shows the accuracies obtained by using segmentation results. Two segmentation algorithms, Susaki (Susaki, 2012a) and ENVI EX 4.8, were compared. 


\section{Discussion}

\subsection{Effects of Horizontal Shift}

Absolute orientation could not be applied to the images at $t_{2}$ which were acquired one day after the disaster. Even when the absolute orientation was implemented, horizontal shifts caused by the earthquake remained (Figures 4(a) and 4(b)). Figures 13 and 14 show that the accuracies were independent of horizontal shifts for Study Area 1, but they were dependent for Study Area 2. This may depend on the mixture of damaged and undamaged buildings. Study Area 1, which is a flat area, lost almost all of buildings. On the other hand, Study Area 2, which is partly flat and partly hilly, lost almost all of buildings in the flat area, but lost no building in the hilly area. Figure 8(f) shows that if no horizontal shift is considered, more commission errors were found in the hilly area. This is because height changes were examined for falsely matched buildings, and so they were falsely classified as "damaged" buildings. Therefore, horizontal shift should be considered in damage examination so that the method can be applied to any damaged areas.

Automatic detection of horizontal shifts using SIFT performed as well as manual detection (Figures 13 and 14). It was found that manually detected local shifts did not give the best accuracy. In the experiments, we assigned local shifts to $200 \mathrm{~m}$ x $200 \mathrm{~m}$ grids. But, this grid size may not be optimal. On the other hand, the average of the local shifts loses local features but generates a more stable estimation. According to the results shown in Figures 13 and 14, using the average of the local shifts obtained by SIFT is acceptable for extracting damaged buildings.

The horizontal shifts caused by earthquake and a lack of absolute orientation may not be constant through an image. To adjust for this fact, our method uses a search window to find a locally optimal point in the post-disaster image corresponding to a pre-disaster pixel. Figure 6 shows that with GIS data, a $1 \times 1$ search window (no search) generated the best accuracies for both number and area. It also shows that without GIS data, there is no significant relation between a searching window size and accuracy for Study Area 1, but that there is a significant relation for Study Area 2. This fact provides an interesting insight: when building boundaries are accurate, there is no need to search for the optimal point; when they are not accurate, because they are from segmentation and filtering results, searching within a certain size of window helps to improve the accuracy. In conclusion, the selection of the optimal search window size depends on the availability of GIS data.

\subsection{DSM Accuracy and Parameter Selection}

Figure 6 shows that for Study Area 2, all the accuracies of the results from DSMs at $t_{1}$ and $t_{3}$ are better than those from DSMs at $t_{1}$ and $t_{2}$. Theoretically, this is consistent with the inclusion of absolute orientation. For Study Area 1 , there is no significant difference between the accuracies of the results from DSMs at $t_{1}$ and $t_{2}$ and the accuracies of the results from DSMs at $t_{1}$ and $t_{3}$. Again, this fact can be explained from the damage in Study Area 1. Because Study Area 1 lost almost all its buildings over the entirety of the area, the effect of the absolute orientation was not clearly found in the results.

As mentioned in Subsection 3.2, the DSM generated by using the same software in another study area had a $0.71 \mathrm{~m}$ standard deviation of the height change between LiDAR data and the DSM (Susaki et al., 2013). The method proposed in this paper uses the height change $\left(T_{2}\right)$ and a ratio parameter $\left(T_{3}\right)$. This ratio parameter 
contributed to eliminating the effects of DSM errors because the two parameters may be relatively more stable than the window size $\left(T_{1}\right)$. F-values of Figure 6 show that the two study areas have different optimal values. We recognize that the optimal parameter values may depend on the accuracy of generated DSM. However, if our method is applied to another study area, the values used in the present study can be applied as defaults.

\subsection{Comparison with Existing Methodologies}

Figures 11 to 14 show that the performance of the method of Tian et al. (2013) was significantly worse than that of the proposed method, especially for Study Area 2. While Study Area 1 lost almost all its buildings, Study Area 2 retains almost all buildings in the hilly areas (north-western area in Figures 1(c) and 1(e)). Because the method of Tian et al. (2013) combines both brightness and height changes into the function of change vector analysis, the results of extracting damaged buildings are sensitive to brightness change. As shown in Figures 12(f) and 12(h), undamaged buildings were falsely classified as damaged mainly due to brightness changes even though the heights showed almost no change. The results for Study Area 1 were similar to those obtained by the proposed method. The main reason may be that almost all buildings were damaged, and the errors caused by brightnessbased evaluation were less than those in Study Area 2. These results clearly show that the proposed method, based on height change only, outperformed the method of Tian et al. (2013).

In terms of segmentation performance, Figure 14 shows that there is no significant difference in accuracies between the algorithm of Susaki (2012a) and ENVI EX for both study areas. Susaki (2012a) reported that the segmentation algorithm outperformed ENVI EX when they were both applied to dense urban areas where there are unclear boundaries between buildings and the shadows cast by neighboring buildings are found on the image. Because the two study areas in the present research are not dense urban areas, this may have led to the performances of the two segmentation algorithms being similar.

\section{Conclusions}

In this paper, we presented a method for extracting individual damaged buildings by using aerial images observed pre- and post-disaster. Using DSMs generated from pre- and post-disaster images, the method finds significant height changes in building regions, which are provided from GIS data or the results of segmentation of pretsunami disaster images and the filtering of pre-disaster DSMs. Horizontal shifts due to land deformation caused by earthquakes and to orientation error are automatically estimated by SIFT. To allow for the errors in the generated DSMs, the proposed method uses three parameters: (1) a window size to locate the new position of the same pixel $\left(T_{1}\right)$; (2) height change $\left(T_{2}\right)$; and (3) minimum percentage of damaged pixels per region $\left(T_{3}\right)$. It was found that while the values of $T_{2}$ and $T_{3}$ are stable, $T_{1}$ is dependent on the data set. Validation with data from before and after the 2011 tsunami showed that the proposed method extracted damaged buildings with high accuracy (94\% to $96 \%$ in number and $96 \%$ to $98 \%$ in area) when GIS data are available and with lower accuracy (69\% to $79 \%$ in area) when GIS data are unavailable. We conclude that our method can automatically generate maps of buildings damaged not only by a tsunami but also by other disasters. 


\section{Acknowledgment}

This research was supported by a Grant-in-Aid for Scientific Research (KAKENHI) for Young Scientists (B) (24760412) from the Ministry of Education, Culture, Sports, Science and Technology, Japan.

\section{References}

Brunner, D., Lemoine, G. and Bruzzone, L., 2010. Earthquake damage assessment of buildings using VHR optical and SAR imagery. IEEE Trans. Geosci. Remote Sens. 48, 2403 - 2420.

Chen, S. W. and Sato, M., 2013. Tsunami damage investigation of built-up areas using multitemporal spaceborne full polarimetric SAR images. IEEE Trans. Geosci. Remote Sens., 51, 1985-1997.

Chini, M., Pulvirenti, L. and Pierdicca, N., 2012. Analysis and interpretation of the COSMO-SkyMed observations of the 2011 Japan Tsunami. IEEE Trans. Geosci. Remote Sens., 9, 467-471.

Chini, M., Piscini, A., Cinti, F. R., Amici, S., Nappi, R. and Martini, P. M. D., 2013. The 2011 Tohoku (Japan) Tsunami inundation and liquefaction investigated through optical, thermal, and SAR data. IEEE Geosci. Remote Sens. Letters, 10, 347-351.

Dekker, R. J., 2011. High-resolution radar damage assessment after the earthquake in Haiti on 12 January 2010. IEEE J. of Selected Topics Applied Earth Observations and Remote Sens., 4, 960-970.

ENVI EX, 2009. ENVI EX User's Guide; Available at http://www.exelisvis.com/portals/0/pdfs/enviex/ ENVI_EX_User_Guide.pdf (accessed on Jun. 2, 2013).

Gamba, P., Dell'Acqua, F. and Trianni, G., 2007. Rapid damage detection in the Bam area using multitemporal SAR and exploiting ancillary data, IEEE Trans. Geosci. Remote Sens., 45, 1582-1589.

Geospatial Information Authority of Japan, 2013a. Crustal movement of GPS base stations obtained from GPS measurement, available at http://www.gsi.go.jp/common/000059672.pdf (accessed on Jun. 2, 2013).

Geospatial Information Authority of Japan, 2013b. Crustal movement of GPS base stations obtained from GPS measurement (municipality-base), available at http://www.gsi.go.jp/common/ 000059961.pdf (accessed on Jun. 2, 2013).

Geospatial Information Authority of Japan, 2013c. 50-m DEM, Geospatial Information Authority of Japan, available at http://www.gsi.go.jp/ geoinfo/dmap/dem50m-index.html (accessed on Jun. 2, 2013).

Headquarters for Earthquake Research Promotion, 2013. Probability of earthquake occurrence on a long-term forecast. available at http://www.jishin.go.jp/main/choukihyoka/kaikou.htm (accessed on Jun. 2, 2013).

Inpho, 2013a. Match-AT DSM, Inpho, available at http://www.inpho.de/index.php?seite= index_match-at (accessed on Jun. 2, 2013).

Inpho, 2013b. Match-T DSM, available at http://www.inpho.de/index.php?seite=index_match-t (accessed on Jun. 2, 2013).

Jin, X., 2009. Segmentation-based image processing system. US Patent 20,090,123,070, filed 14 November 2007, and issued 14 May 2009.

Khoshelham, K., Elberink, S.O. and Xu, S., 2013. Segment-based classification of damaged building roofs in aerial laser scanning data, IEEE Geoscic. Remote Sens. Letters, 10, 1258-1262. 
Li, X., Guo, H., Zhang, L., Chen, X. and Liang, L., 2012. A new approach to collapsed building extraction using RADARSAT-2 polarimetric SAR imagery. IEEE Geosci. Remote Sens. Letters, 9, 677-681.

Liu, W. and Yamazaki, F., 2011. Urban monitoring and change detection of central Tokyo using high-resolution X-band SAR images. Proc. IEEE Int. Geosci. Remote Sens. Symp., Vancouver, BC, Canada, 2133-2136.

Liu, W., Yamazaki, F., Gokon, H. and Koshimura, S., 2012. Extraction of damaged buildings due to the 2011 Tohoku, Japan Earthquake Tsunami. Proc. IEEE Int. Geosci. Remote Sens. Symp., Munich, Germany, 4038-4041.

Lowe, D., 2004. Distinctive image features from scale-invariant keypoints. International Journal of Computer Vision, 60(2), 91-110.

Matsuoka, M. and Yamazaki, F., 2004. Use of satellite SAR intensity imagery for detecting building areas damaged due to earthquakes. Earthquake Spectra, 20, 975-994.

Mongus, D. and Žalik, B., 2012. Parameter-free ground filtering of LiDAR data for automatic DTM generation. ISPRS J. Photogramm., 67, 1-12.

Novack, T., Esch, T., Kux, H. and Stilla, U., 2011. Machine learning comparison between WorldView-2 and QuickBird-2-simulated imagery regarding object-based urban land cover classification. Remote Sens., 3, 2263-2282.

Pesci, A., Teza, G., Bonali, E., Casula, G. and Boschi, E., 2013. A laser scanning-based method for fast estimation of seismic-induced building deformations. ISPRS J. Photogram. Remote Sens., 79, 185-198.

Sato, M. and Chen, S. W., 2012. Polarimetric SAR analysis of Tsunami damage following the March 11, 2011 East Japan Earthquake. Proceedings of the IEEE, 100, 2861-2875.

Sithole, G. and Vosselman, G., 2005. Filtering of airborne laser scanner data based on segmented point clouds. Int. Arch. Photogram. Remote Sens. Spat. Inform. Sci, 36 (Part 3/W19), 66-71.

Susaki, J., 2012a. Segmentation of shadowed buildings in dense urban areas from aerial photographs. Remote Sens., 4, 911-933.

Susaki, J. 2012b. Adaptive slope filtering of airborne LiDAR data in urban areas for digital terrain model (DTM) Generation, Remote Sensing, 4, 1804-1819.

Susaki, J., Komiya, Y. and Takahashi, K., 2013. Calculation of enclosure index for assessing urban landscapes using digital surface models, IEEE J. of Selected Topics Applied Earth Observations and Remote Sens., in press.

Tian, J., Reinartz, P., d’Angelo, P. and Ehlers, M., 2013. Region-based automatic building and forest change detection on Cartosat-1 stereo imagery. ISPRS J. Photogram. Remote Sens., 79, 226-239.

Tong, X., Hong, Z., Liu, S., Zhang, X., Xie, H., Li, Z., Yang, S., Wang, W. and Bao, F., 2012. Building-damage detection using pre- and post-seismic high-resolution satellite stereo imagery: A case study of the May 2008 Wenchuan earthquake, ISPRS J. Photogram. Remote Sens., 68, 13-27.

Tsutsui, K., Rokugawa, S., Nakagawa, H., Miyazaki, S., Cheng, C. T., Shiraishi, T. and Yang, S. D., 2007. Detection and volume estimation of large-scale landslides based on elevation-change analysis using DEMs extracted from high-resolution satellite stereo imagery, IEEE Trans. Geosci. Remote Sens., 45, 1681-1696. 
446 Tuia, D., Pacifici, F., Kanevski, M. and Emery, W. J., 2009. Classification of very high spatial resolution imagery using mathematical morphology and support vector machines, IEEE Trans. Geosci. Remote Sens., 47, 38663879.

449 Watanabe, M., Motohka, T., Miyagi, Y., Yonezawa, C. and Shimada, M., 2012. Analysis of urban areas affected by the 2011 Off the Pacific Coast of Tohoku Earthquake and Tsunami with L-band SAR full-polarimetric mode. IEEE Geosci. Remote Sens. Letters, 9, 472-476.

Wang, T. L. and Jin, Y. Q., 2012. Postearthquake building damage assessment using multi-mutual information from pre-event optical image and postevent SAR image. IEEE Geoscience and Remote Sensing Letters, 9, 452-456.

Yamaguchi, Y., Sato, A., Boerner, W., Sato, R. and Yamada, H., 2011. Four-component scattering power decomposition with rotation of coherency matrix. IEEE Trans. Geosci. Remote Sens., 49, 2251-2258. Proceedings of the IEEE, 100, 2851-2860.

Yonezawa, C. and Takeuchi, S., 2001. Decorrelation of SAR data by urban damages caused by the 1995 460 Hyogoken-Nanbu earthquake. Int. J. Remote Sens., 22, 1585-1600. 
Figure captions:

Figure 1. Aerial images. (a), (b) and (c) Aerial images of Study Area 1 taken on May 15, $2009\left(t_{1}\right)$, March 12, $2011\left(t_{2}\right)$, and May 26, $2011\left(t_{3}\right)$, respectively. Images (a), (b) and (c) were taken using a UCX camera. (d), (e) and (f) Aerial images of Study Area 2 taken on May 18, $2009\left(t_{1}\right)$, March 12, $2011\left(t_{2}\right)$, and May 25, $2011\left(t_{3}\right)$, respectively. Images (d), (e) and (f) were taken using UCX, UCD and RC-30 cameras, respectively.

Figure 2. Flowchart of the proposed method.

Figure 3. Height data. (a), (b) and (c) DSMs of Study Area $1 \mathrm{t}$ at May 15, $2009\left(t_{1}\right)$, March 12, $2011\left(t_{2}\right)$, and May 26, $2011\left(t_{3}\right)$, respectively. (d), (e) and (f) DSMs of Study Area 2 at May 18, $2009\left(t_{1}\right)$, March 12, $2011\left(t_{2}\right)$, and May 25, $2011\left(t_{3}\right)$, respectively.

Figure 4. Horizontal shifts post-tsunami. Shifts of samples for Study Areas (a) 1 and (b) 2. Average shifts between $t_{1}$ and $t_{2}$ and between $t_{1}$ and $t_{3}$ for (a) were $(0.60 \mathrm{~m},-0.27 \mathrm{~m})$ and $(1.10 \mathrm{~m},-1.97 \mathrm{~m})$, respectively. Those for (b) were $(2.57 \mathrm{~m},-1.17 \mathrm{~m})$ and $(4.87 \mathrm{~m},-1.27 \mathrm{~m})$, respectively. The origin of the coordinate system is at bottom left of the images.

Figure 5. Height changes post-tsunami. Height difference between $t_{1}$ and $t_{3}$ against height at $t_{1}$ (a) for damaged buildings, and (b) for undamaged buildings.

Figure 6. Verification of results for the extraction of damaged buildings for various sizes of searching window. $\Delta h$ and $\alpha$ were set to $2.0 \mathrm{~m}$ and 0.5 , respectively. (a) Number of damaged buildings with GIS data, (b) area of damaged buildings with GIS data, and (c) area of damaged buildings with segmentation results obtained by using the method of Susaki (2012a).

Figure 7. Damaged buildings extracted for Study Area 1 by using DSMs at $t_{1}$ and $t_{2}$ with GIS data. (a), (c) and (e) Damaged buildings extracted using (a) the average horizontal shift from SIFT, (c) manual local horizontal shift, and (e) no shift. (b), (d) and (e) Validation results for (a), (c) and (e), respectively. Red denotes true positive, yellow denotes false positive, green denotes false negative and blue denotes true negative.

Figure 8. Damaged buildings extracted for Study Area 2 by using DSMs at $t_{1}$ and $t_{2}$ with GIS data. See the caption of Figure 7 for explanations of the sub-figures.

Figure 9. Building regions generated by segmentation and filtering. (a) and (b) Aerial images of Study Area 1 taken on May 15, 2009, and Study Area 2 taken on May 18, 2009. (c) and (d) Segmentation results obtained by using the method of Susaki (2012a). (e) and (f) Regions generated by masking the segmentation results with 
503 Figure 10. Damaged buildings extracted for Study Area 1 by using DSMs at $t_{1}$ and $t_{2}$ with segmentation results.

504 Damaged buildings extracted using (a) the average horizontal shift from SIFT, (c) manual local horizontal shift, 505 and (e) no shift. (b), (d) and (e) Validation results for (a), (c) and (e), respectively. See the caption of Figure 7 for 506 an explanation of the colors.

Figure 11. Damaged buildings extracted for Study Area 2 by using DSMs at $t_{1}$ and $t_{2}$ with segmentation results. Damaged buildings extracted using (a) the average horizontal shift from SIFT, (c) manual local horizontal shift, and (e) no shift. (b)(d)(e) Validation results for (a), (c) and (e), respectively. See the caption of Figure 7 for an explanation of the colors.

Figure 12. Damaged buildings extracted by using DSMs at $t_{1}$ and $t_{2}$ and the method of Tian et al. (2013). Damaged buildings were extracted using the average horizontal shift from SIFT. (a)(c) Damaged buildings extracted for Study Area 1 using (a) GIS data and (c) segmentation using the method of Susaki (2012a). (b) and (d) Validation results for (a) and (c), respectively. (e)(g) Damaged buildings extracted for Study Area 2 using (e) GIS data and (g) segmentation using the method of Susaki (2012a). (f) and (h) Validation results for (e) and (g), respectively. See the caption of Figure 7 for an explanation of the colors.

Figure 13. Verification of results for the extraction of damaged buildings using GIS data. For the proposed method, a $1 \times 1$ window was used for searching pixels, and $\Delta h$ and $\alpha$ were set to $2.0 \mathrm{~m}$ and 0.5 , respectively. F-value is defined as $2 \times$ precision $\times$ recall $/($ precision + recall $)$ where precision $=\mathrm{TP} /(\mathrm{TP}+\mathrm{FP})$ and recall $=\mathrm{TP} /(\mathrm{TP}+\mathrm{FN})$. (a) Number of damaged buildings, and (b) area of damaged buildings. The method of Tian et al. (2013) was used for comparison.

Figure 14. Verification of results for the extraction of damaged buildings using segmentation results. Two segmentation algorithms were used, a $7 \times 7$ window was used for searching pixels, and $\Delta h$ and $\alpha$ were set to $2.0 \mathrm{~m}$ and 0.5 , respectively. (a) and (b) Areas of damaged buildings for $t_{1}-t_{2}$ and $t_{1}-t_{3}$, respectively. The method of Tian et al. (2013) was used for comparison. 
532 Table captions:

533

534 Table 1. Average horizontal shifts between pre tsunami $\left(t_{1}\right)$ and post tsunami $\left(t_{2}\right.$ or $\left.t_{3}\right)$ for Study Area 1 . 535

536 Table 2. Average horizontal shifts between pre tsunami $\left(t_{1}\right)$ and post tsunami $\left(t_{2}\right.$ or $\left.t_{3}\right)$ for Study Area 2. 537

538 


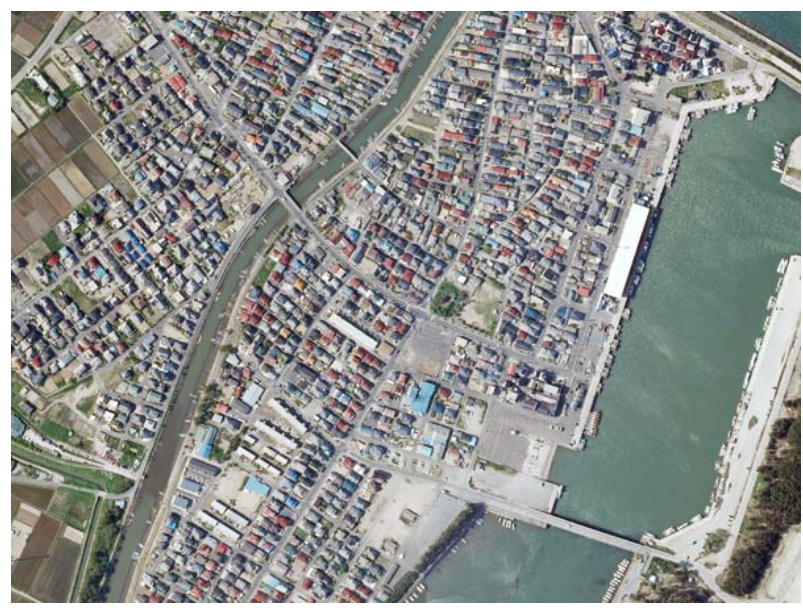

(a)

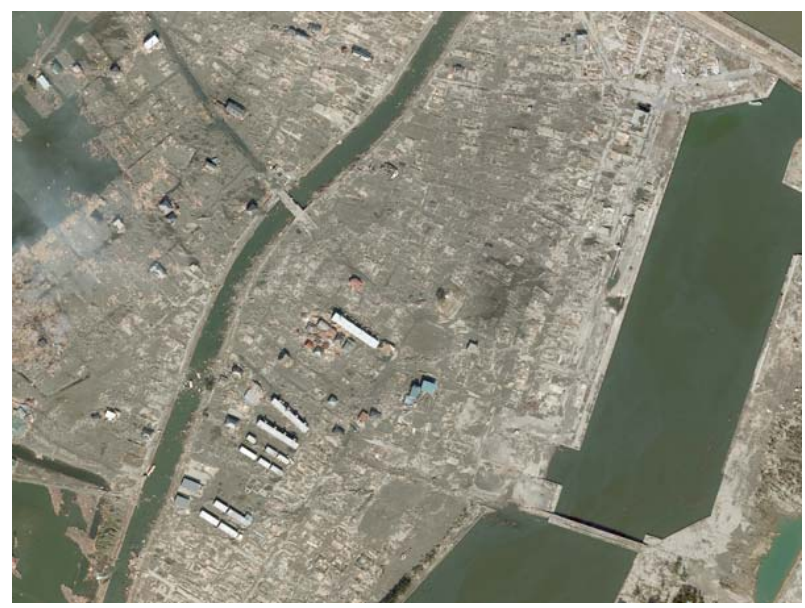

(b)

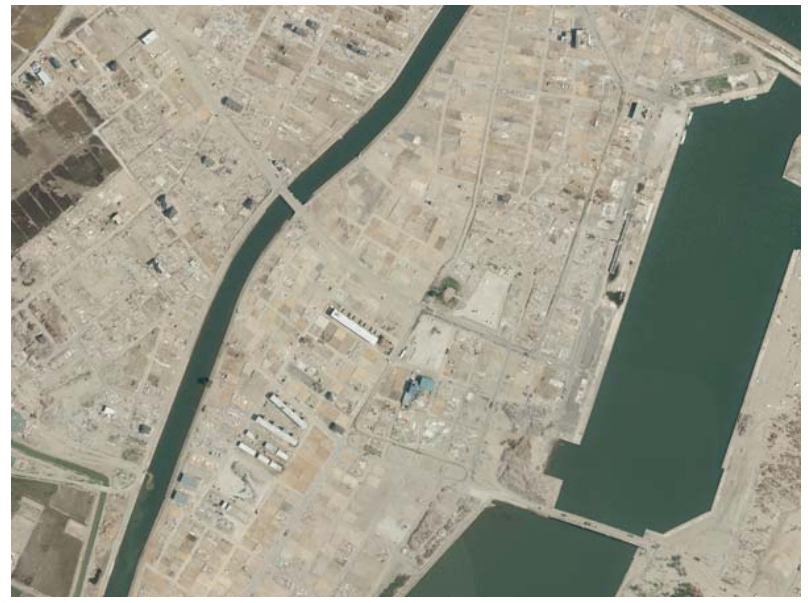

(c)

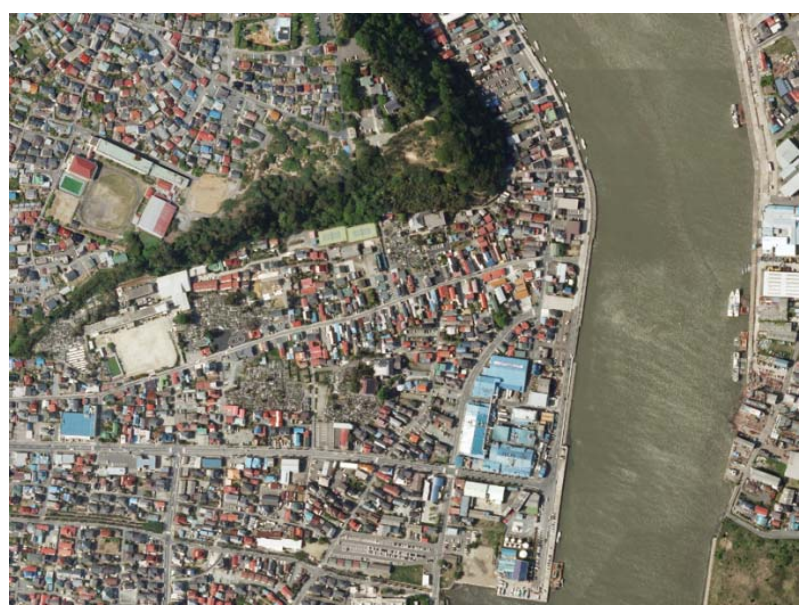

(d)

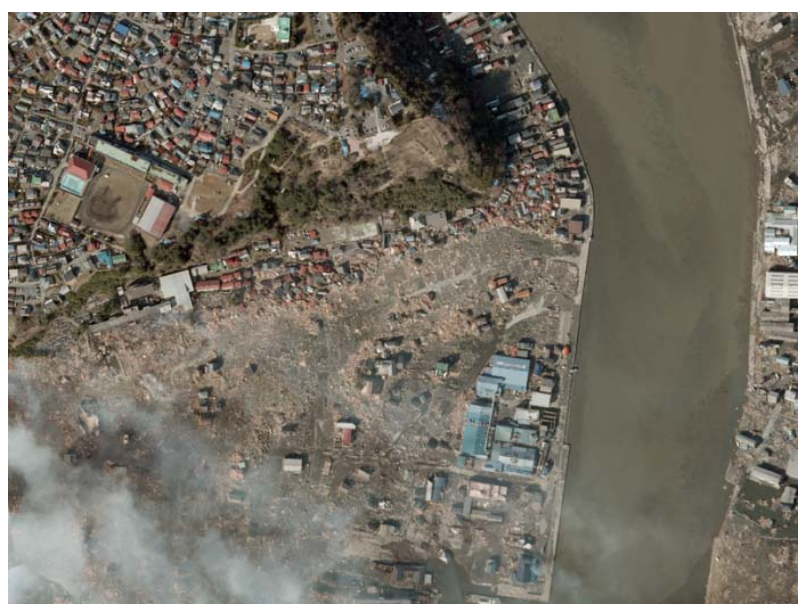

(e)

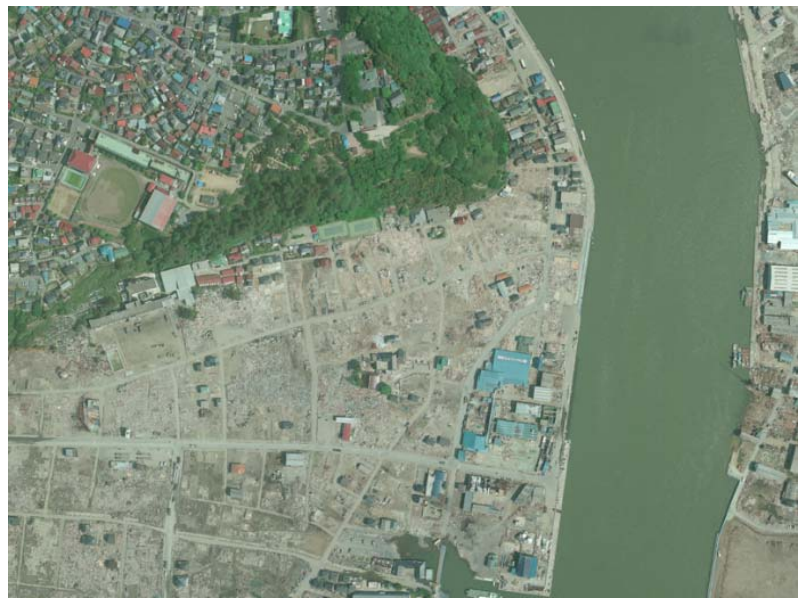

(f)

$\sum_{s}^{N}$

Figure 1. 
(a) GIS data are available

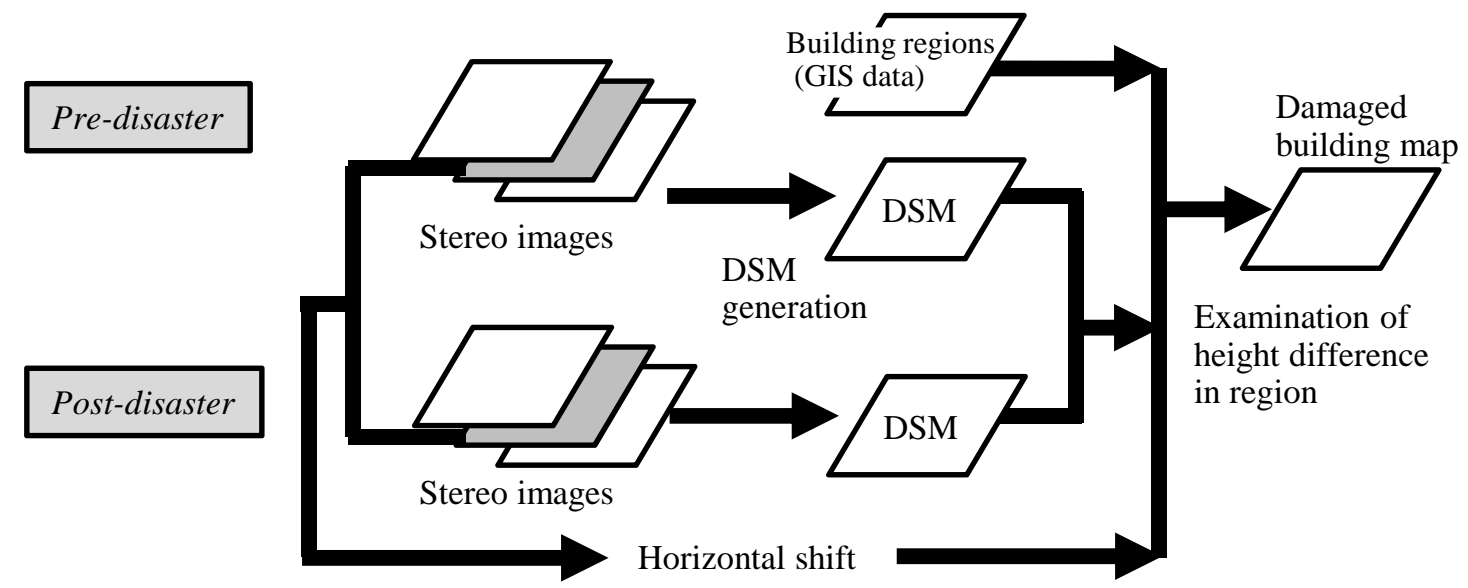

(b) GIS data are not available

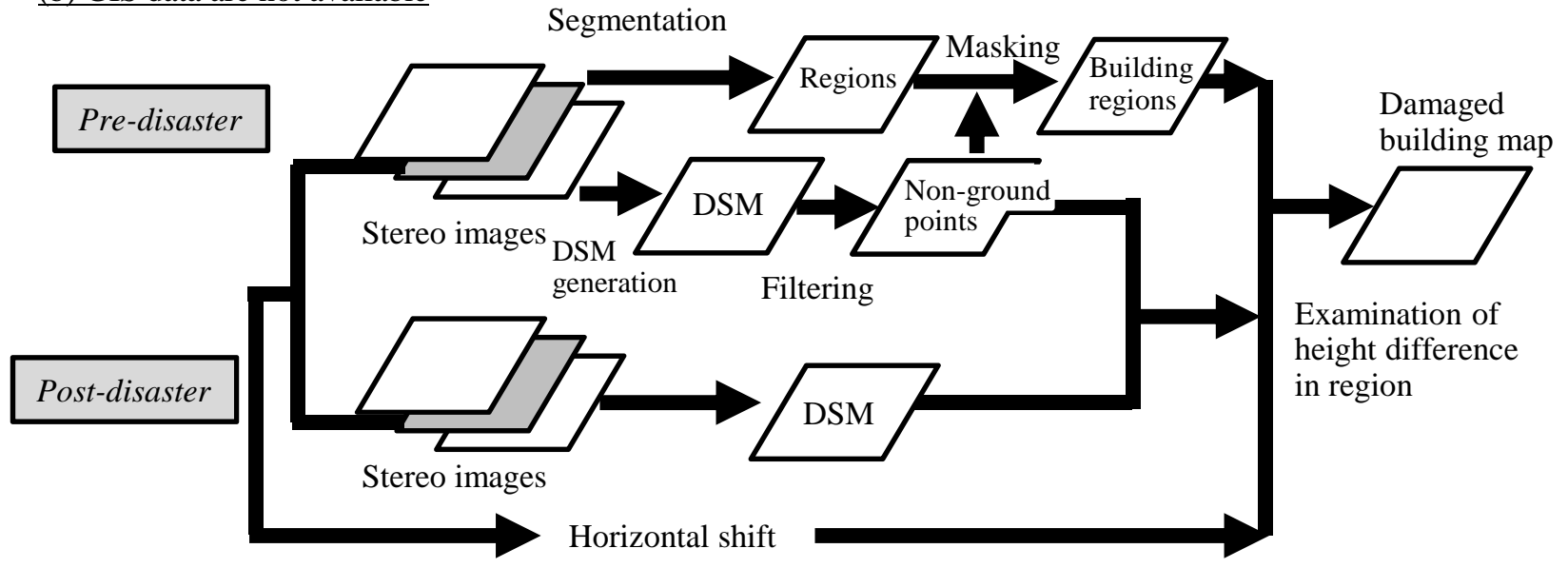

Figure 2. 


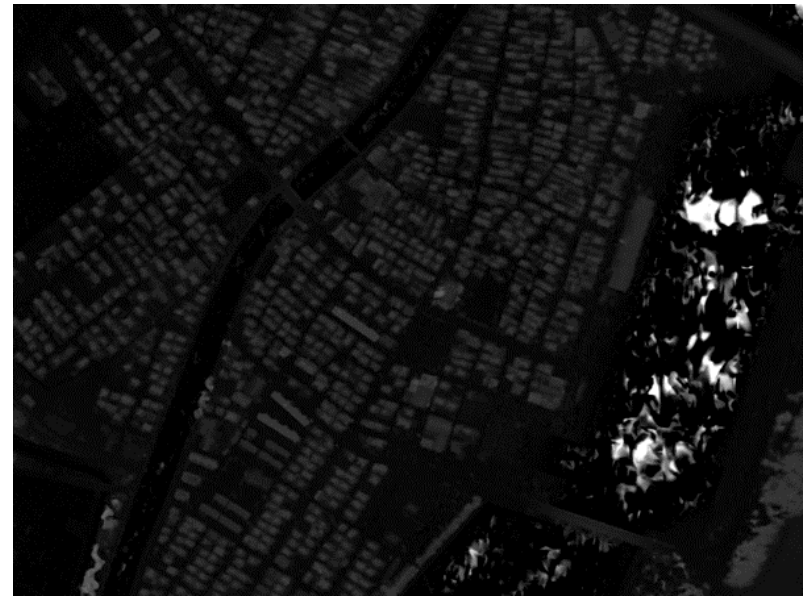

(a)

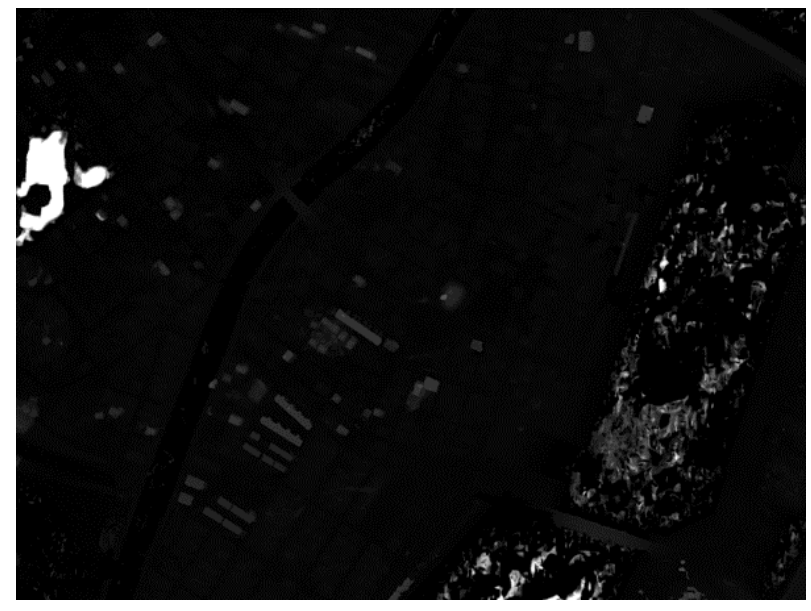

(b)

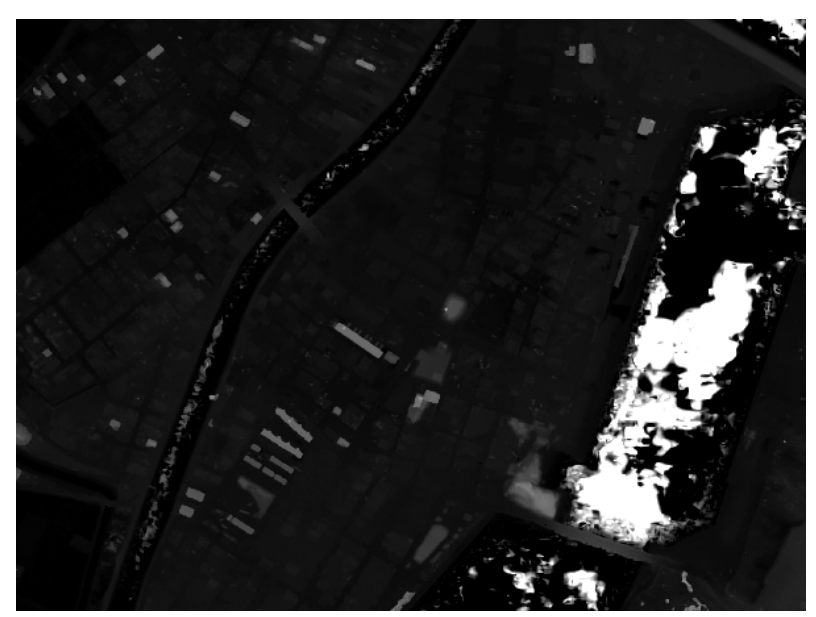

(c)

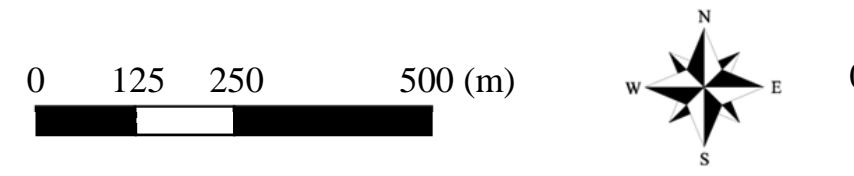

Figure 4.

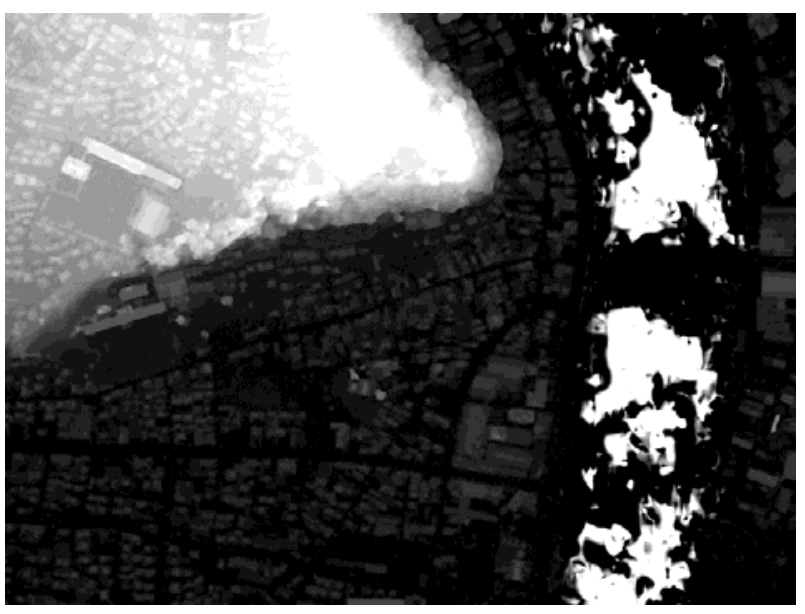

(d)

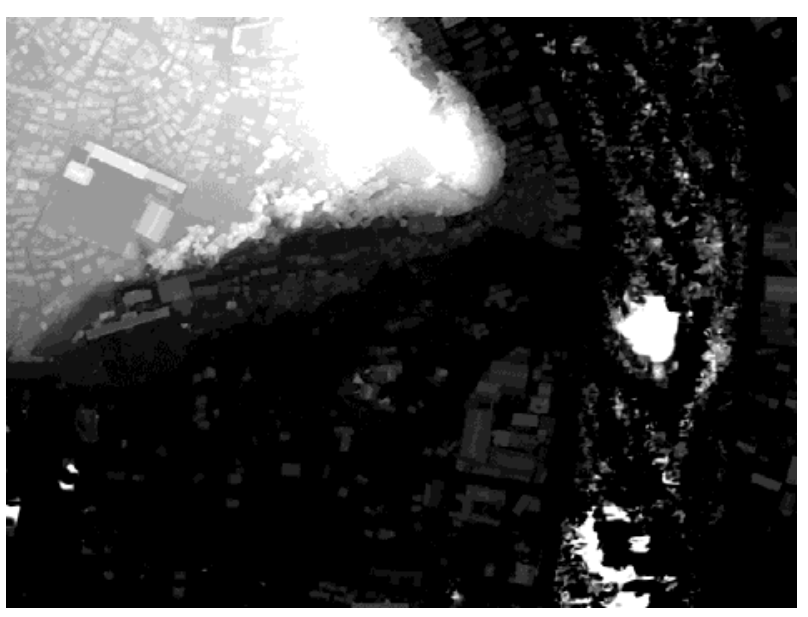

(e)

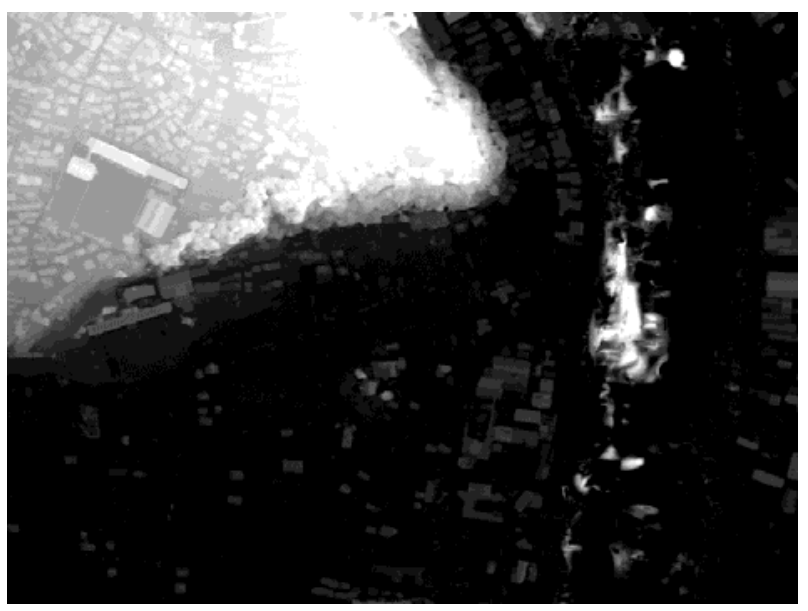

(f)

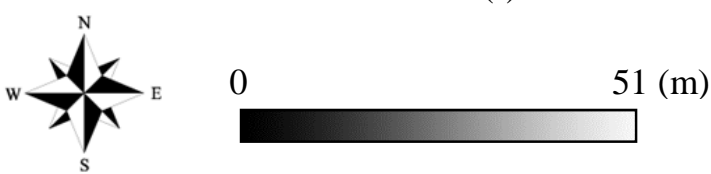




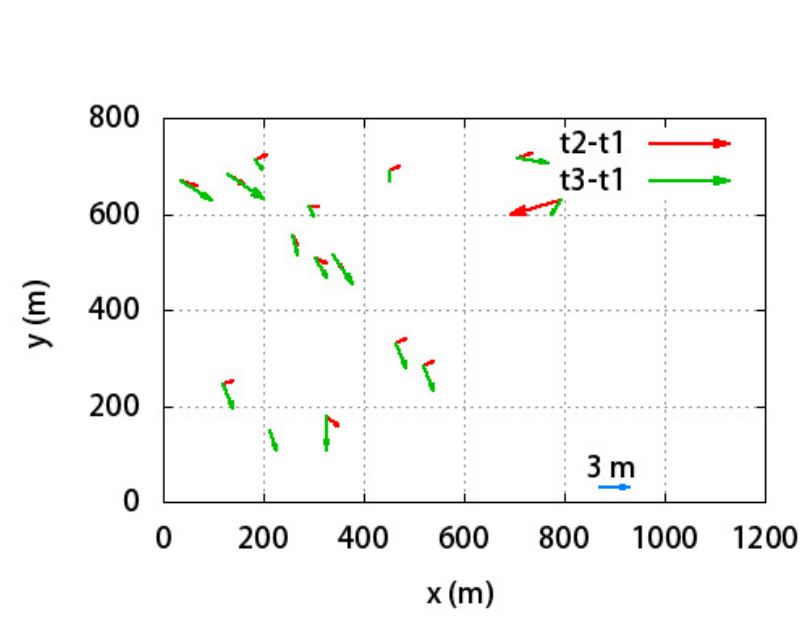

(a)

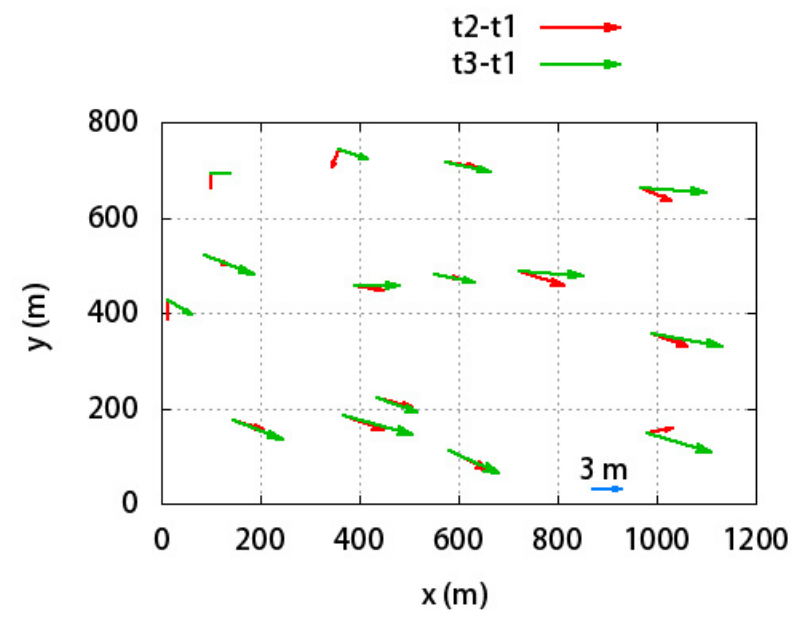

(b)

Figure 4.

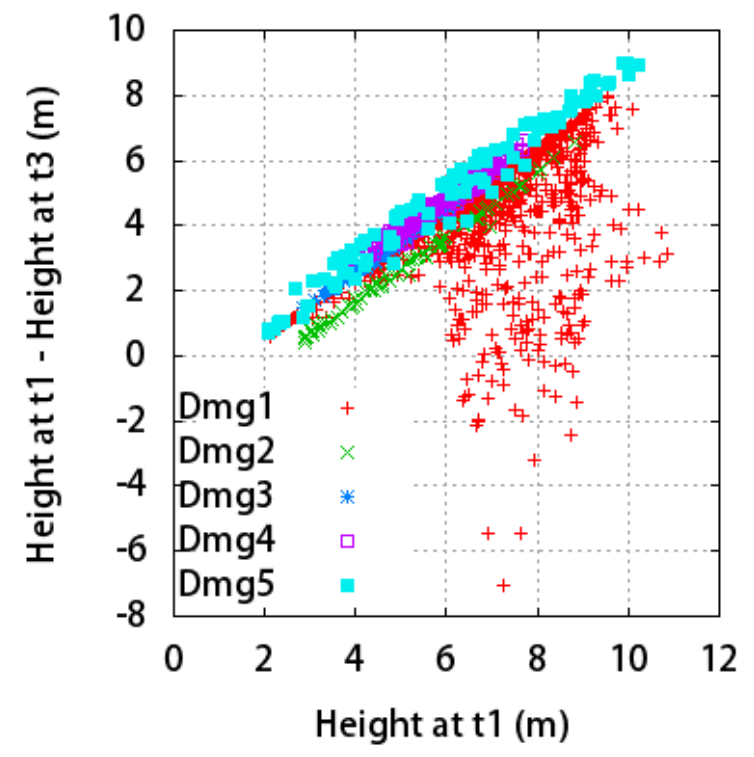

(a)

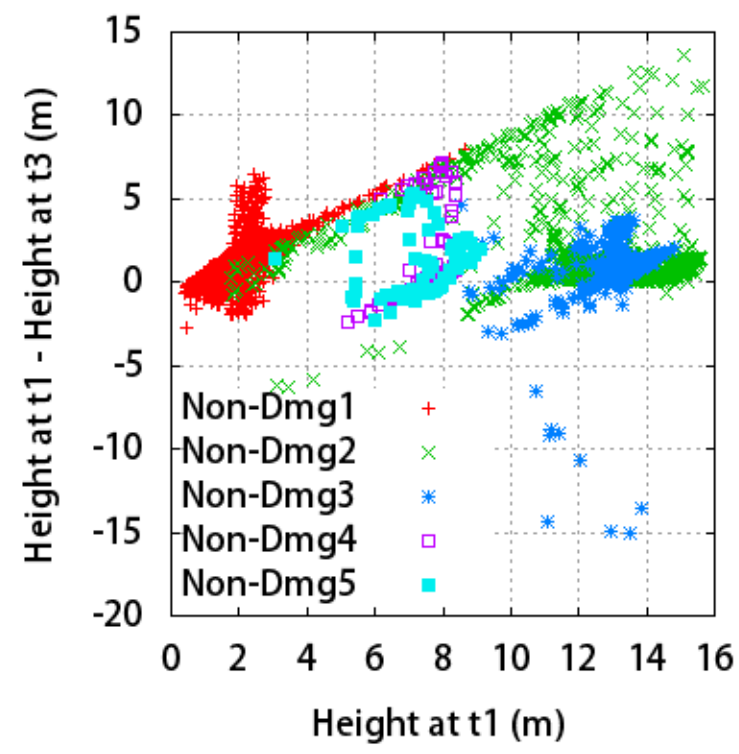

(b)

Figure 5. 


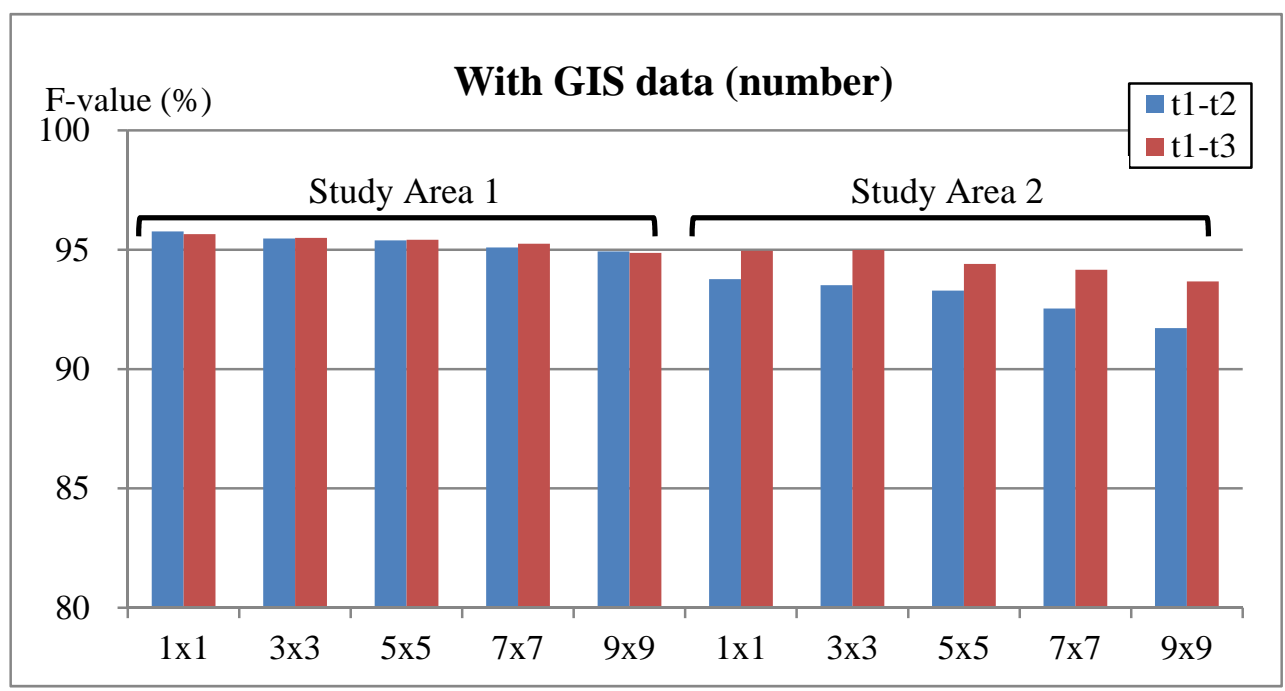

(a)

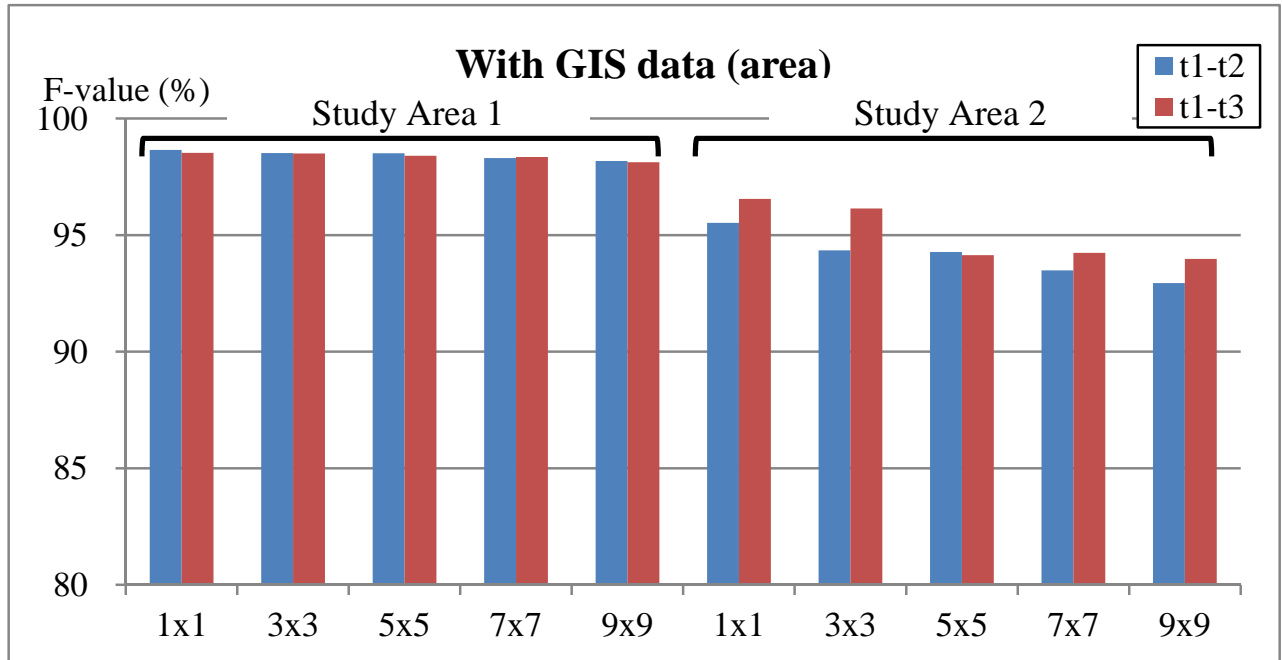

(b)

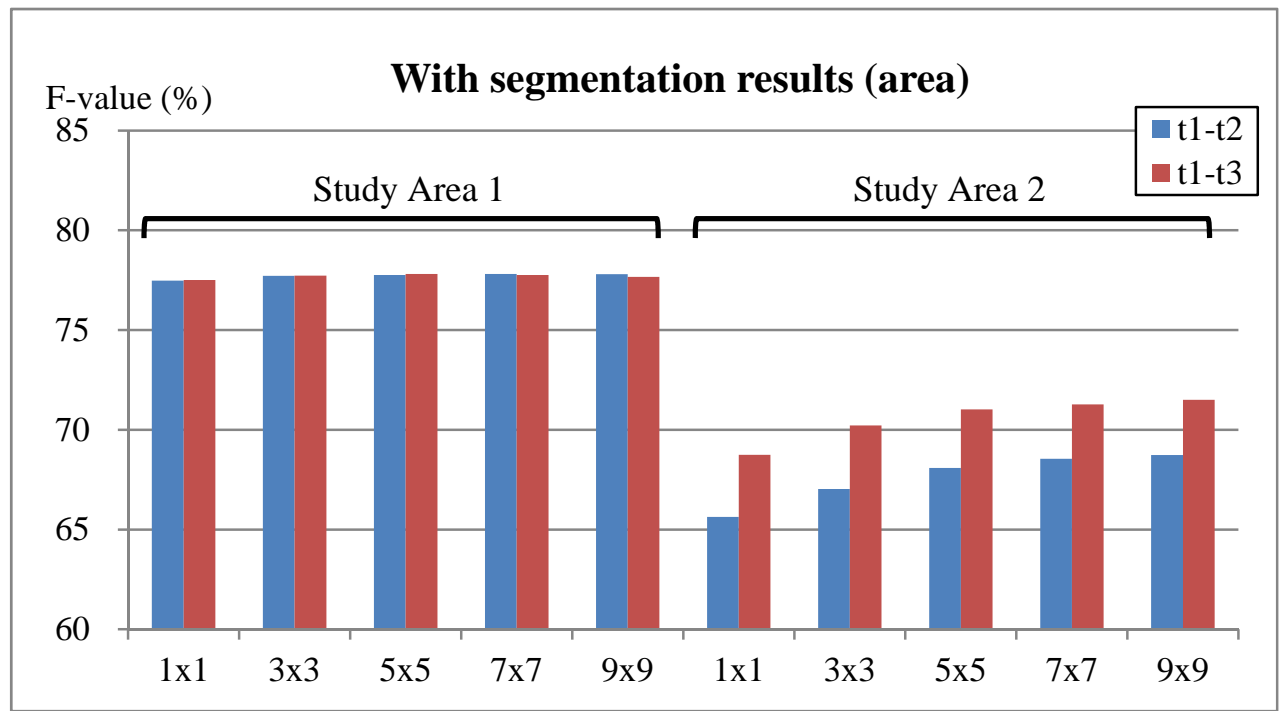

(c)

Figure 6. 


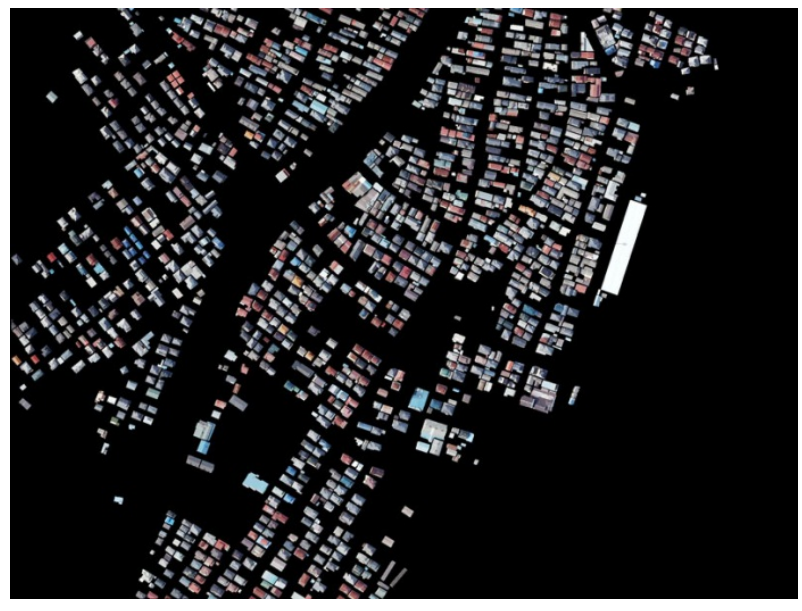

(a)

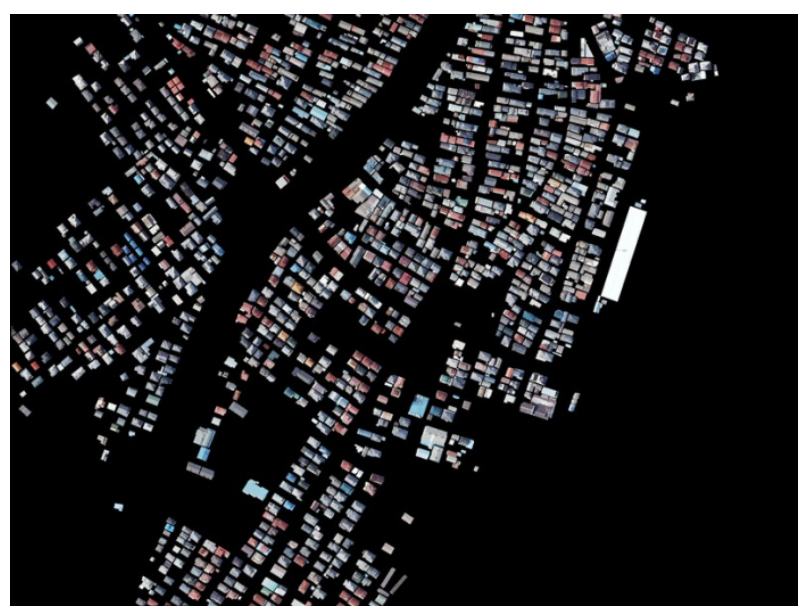

(c)

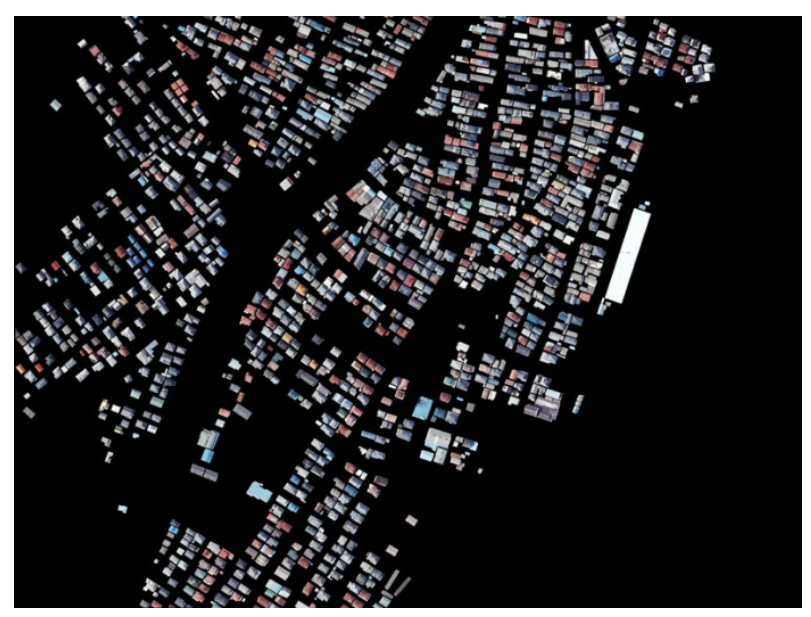

(e)

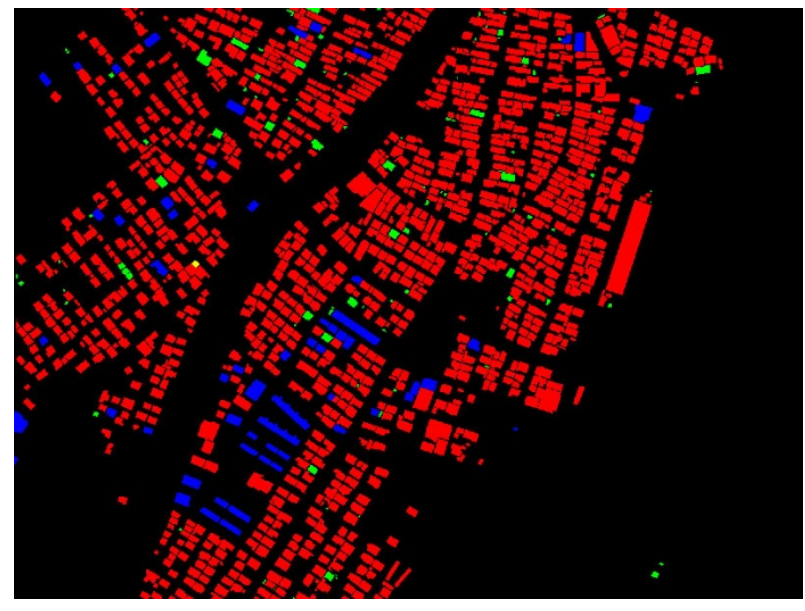

(b)

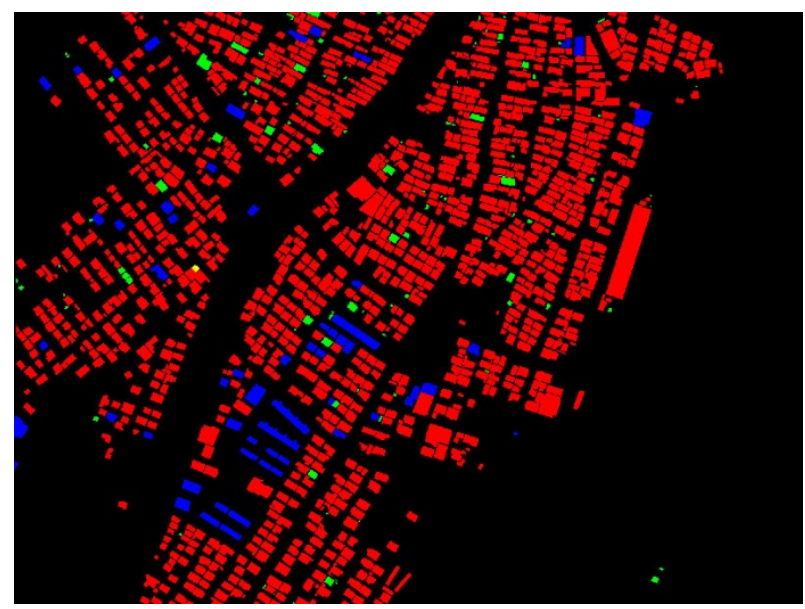

(d)

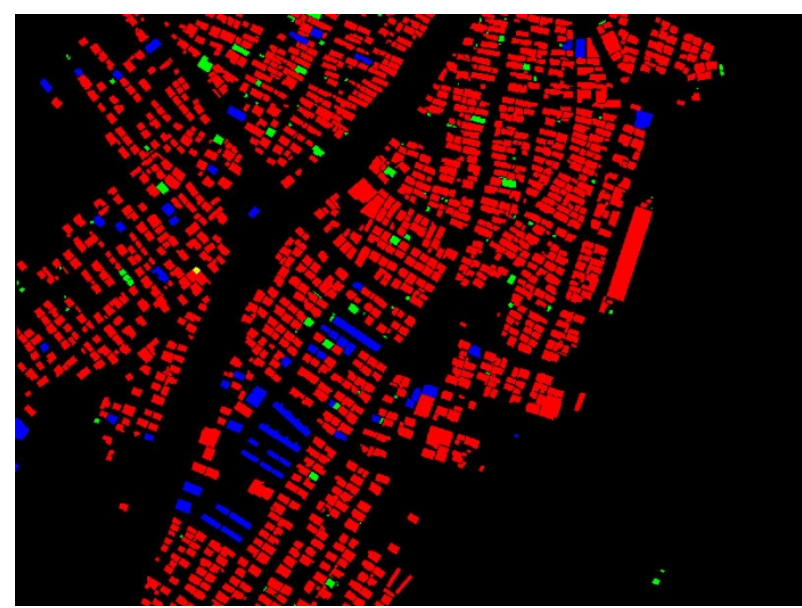

(f)

Figure 7. 


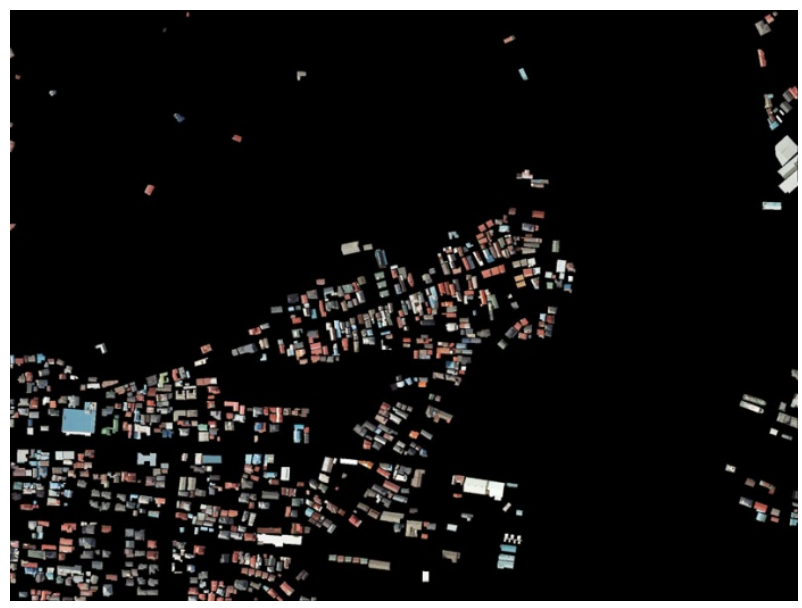

(a)

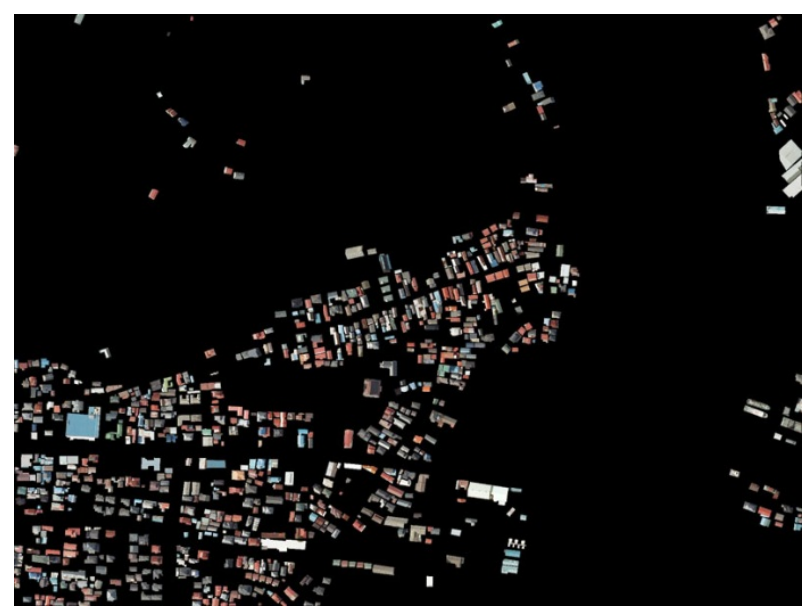

(c)

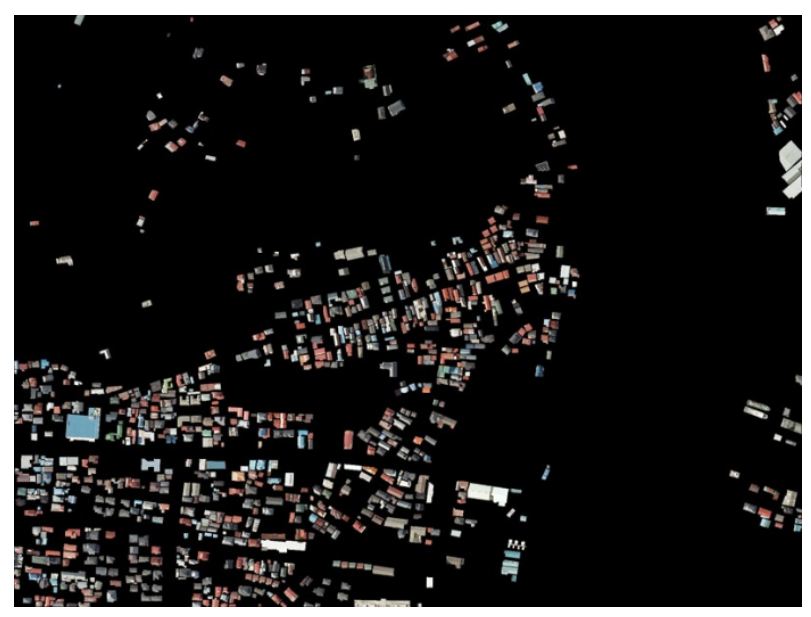

(e)

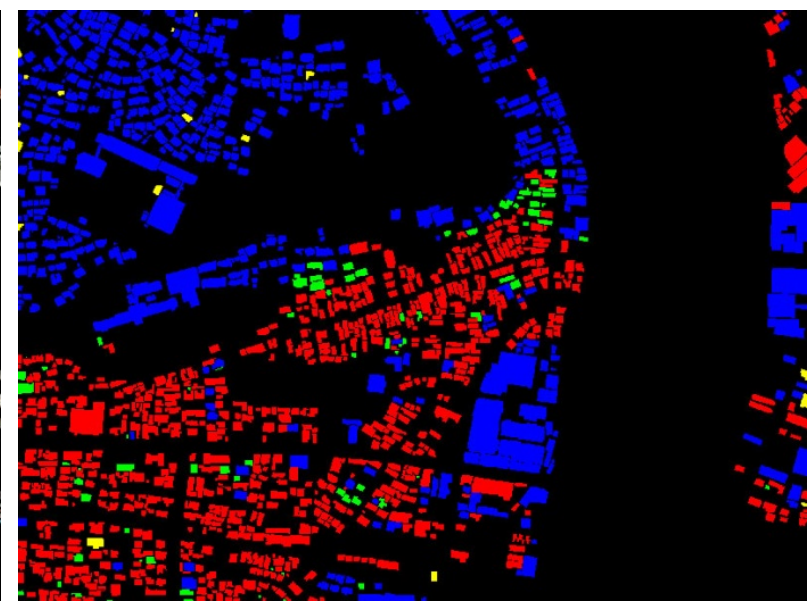

(b)

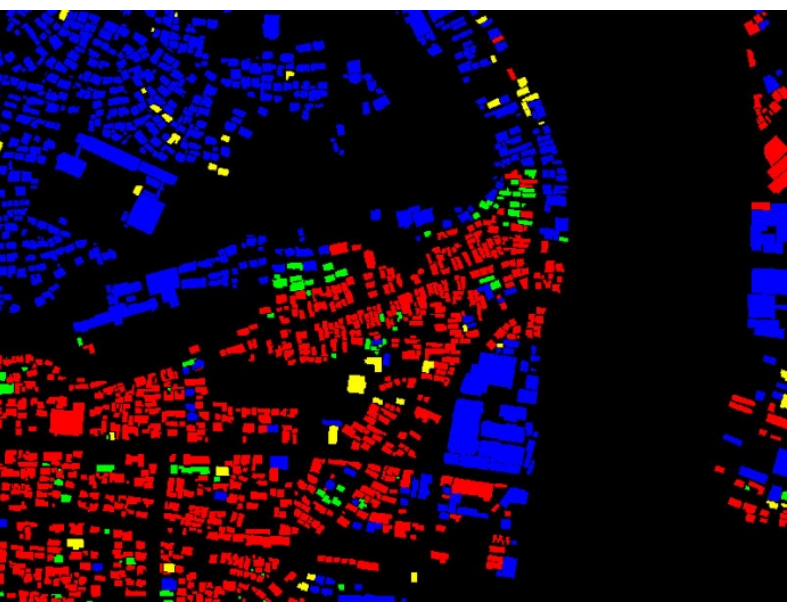

(d)

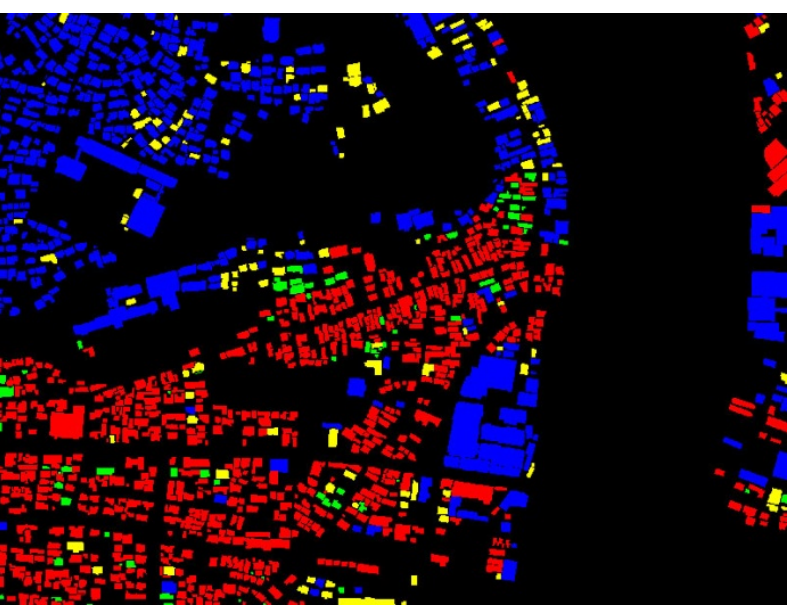

(f)

Figure 8. 


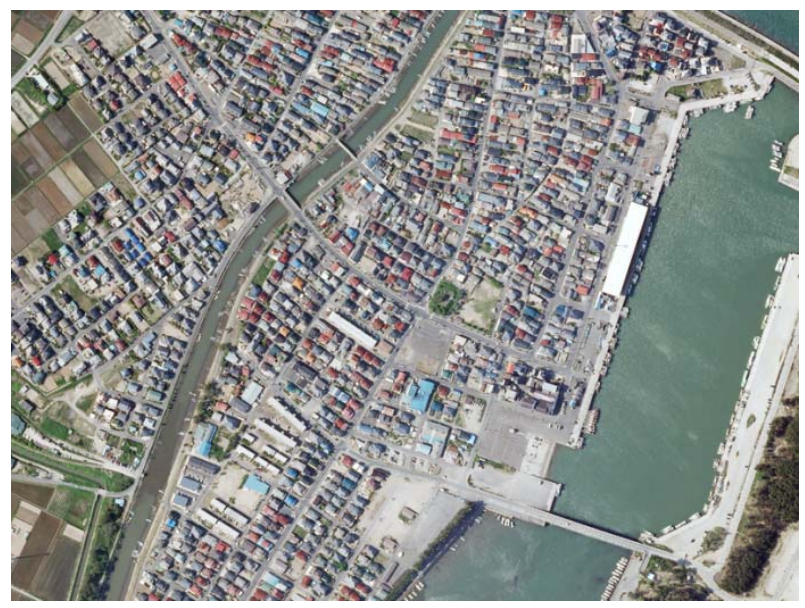

(a)

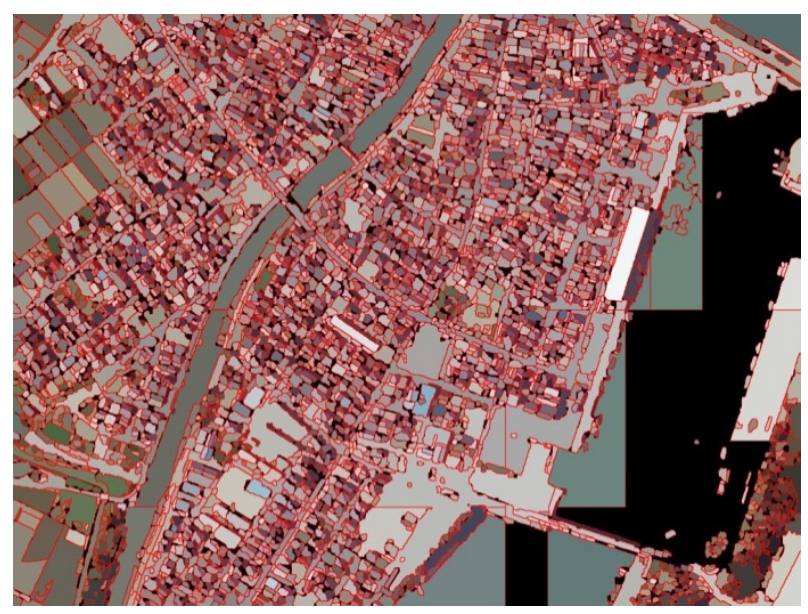

(c)

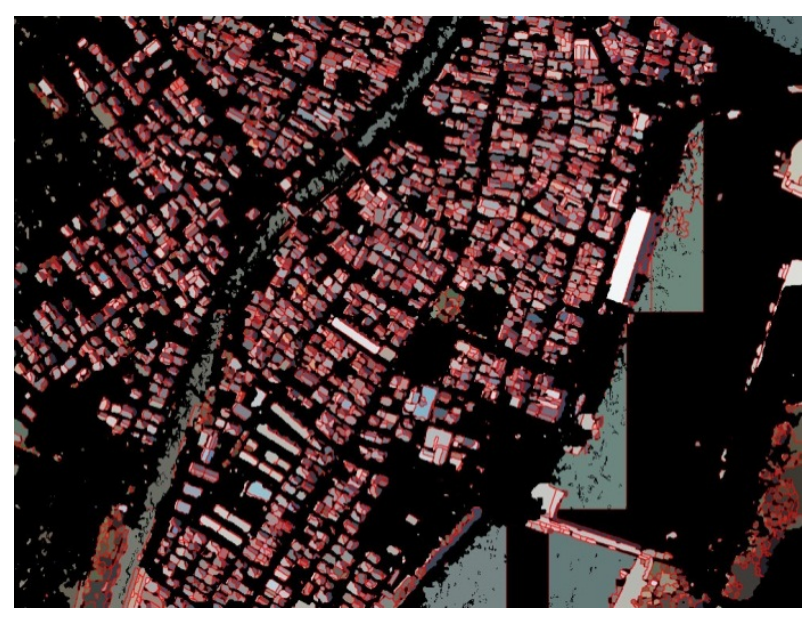

(e)

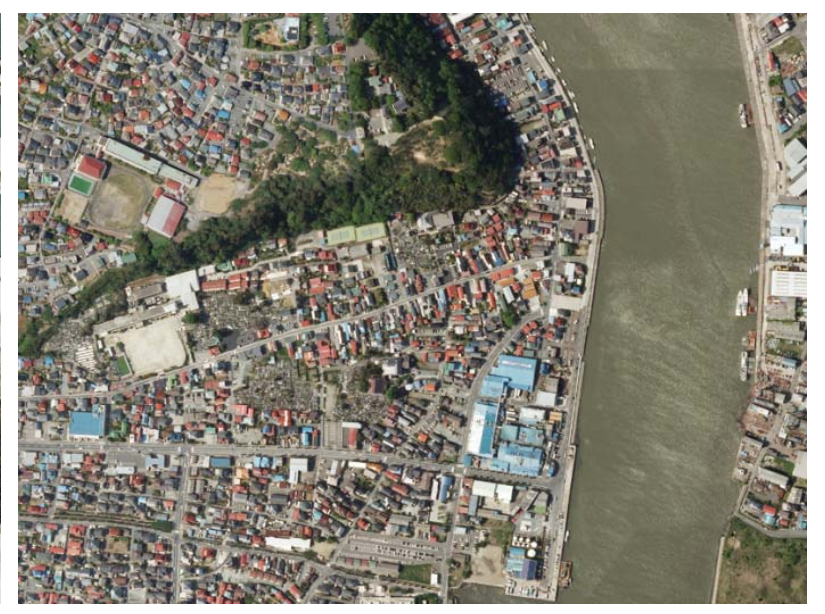

(b)

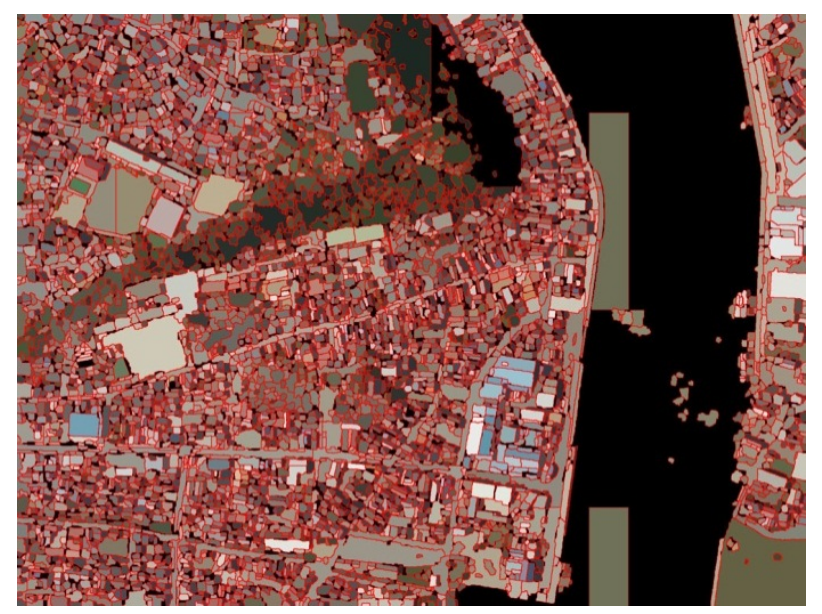

(d)

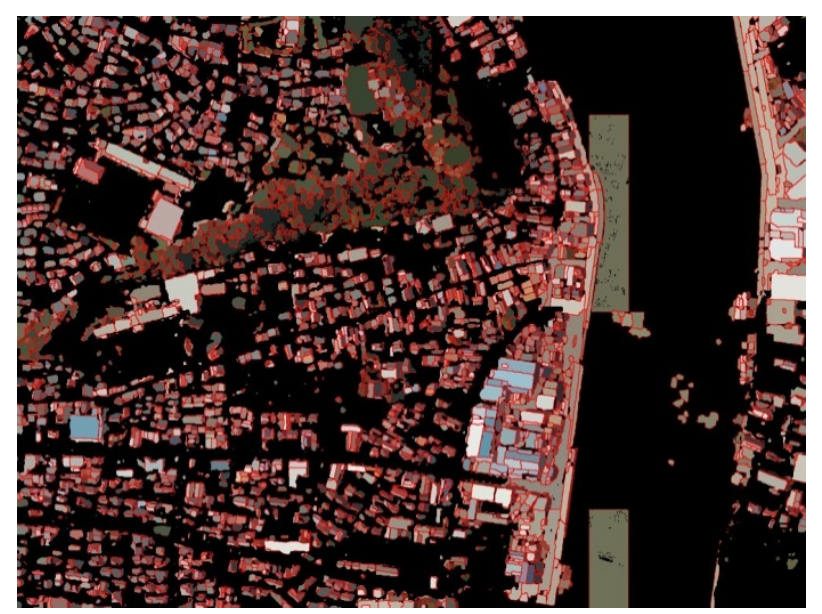

(f)

Figure 9. 


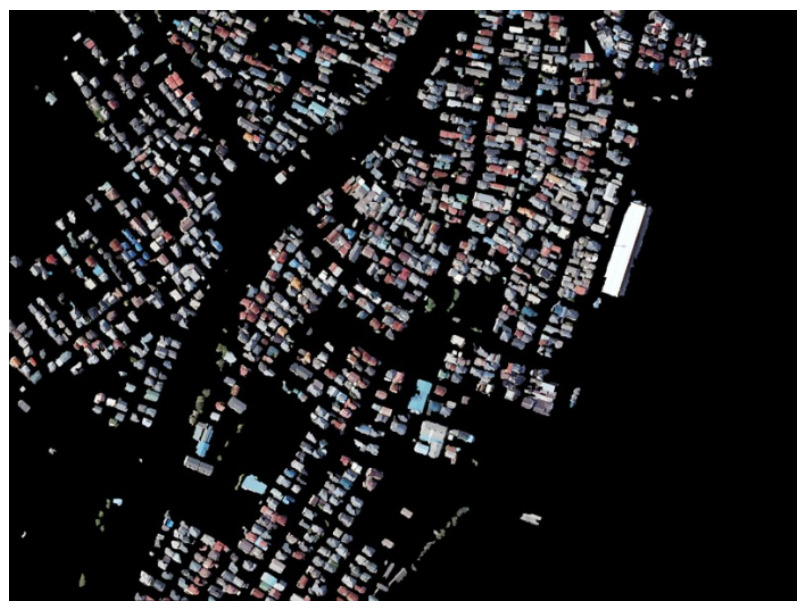

(a)

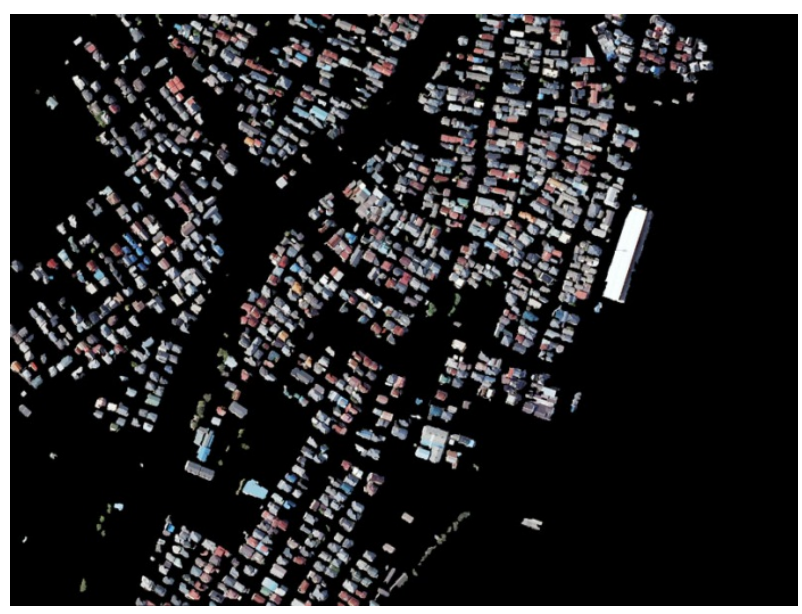

(c)

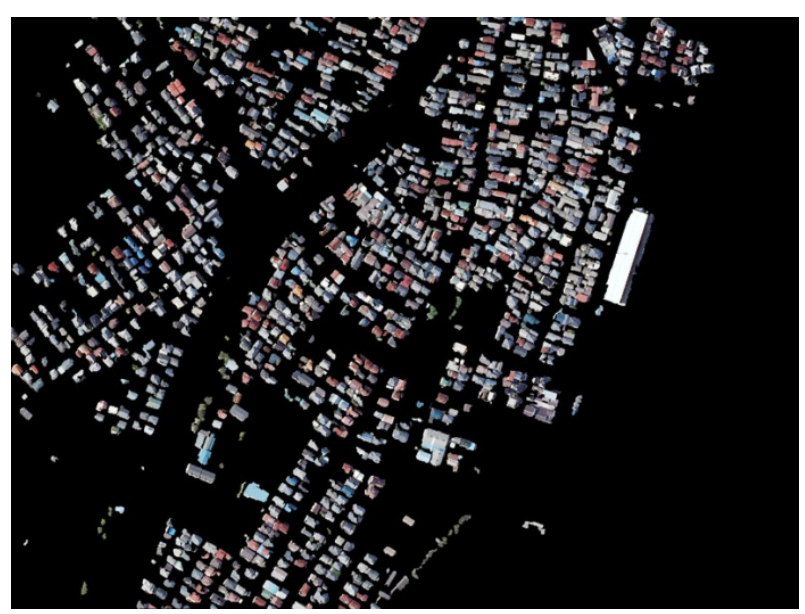

(e)

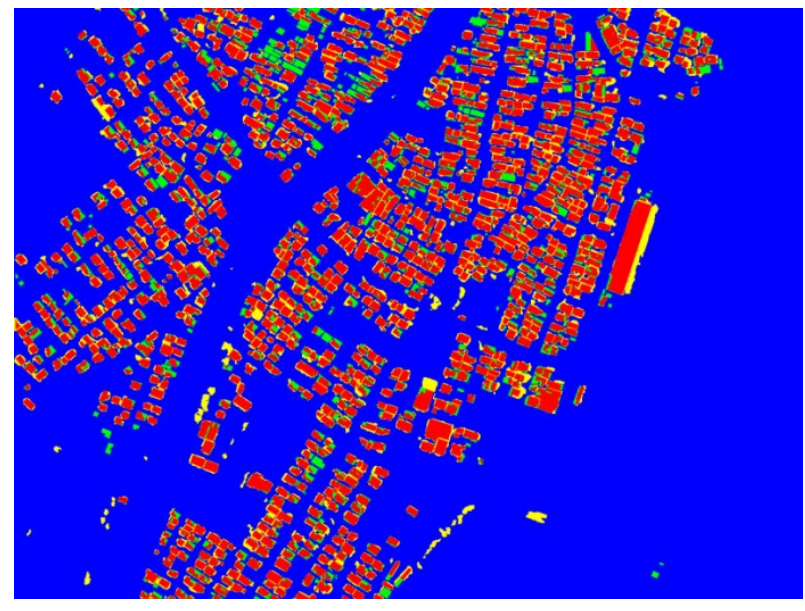

(b)

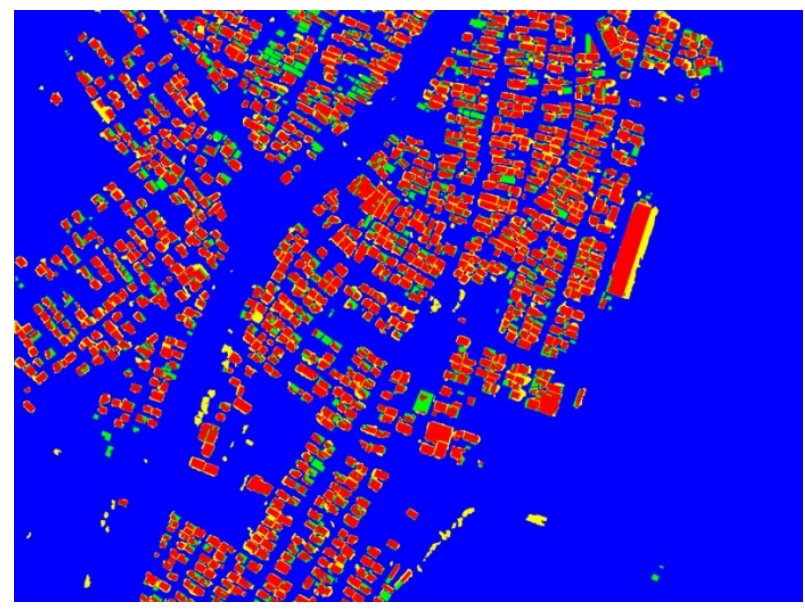

(d)

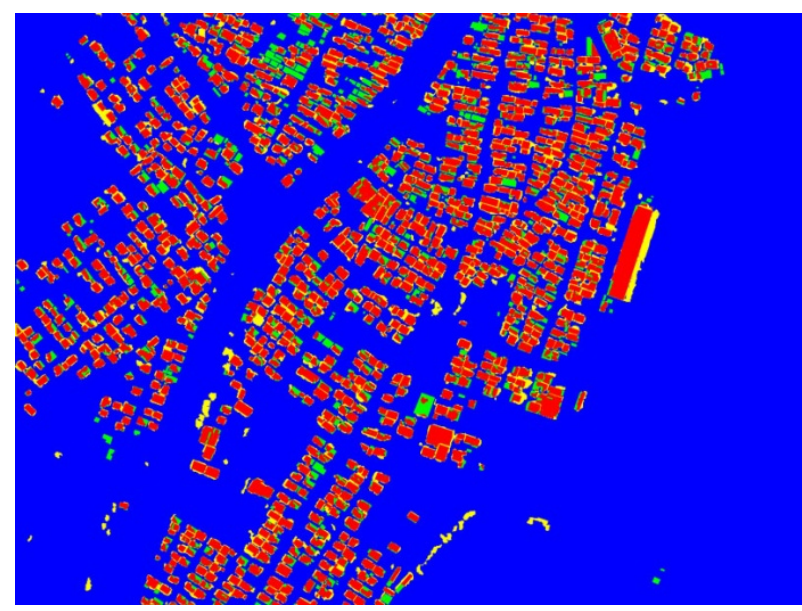

(f)

Figure 10. 


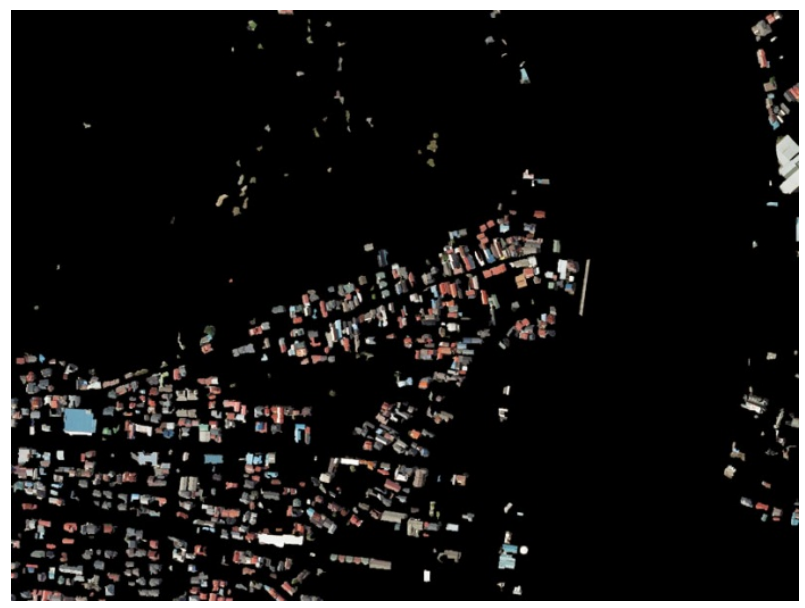

(a)

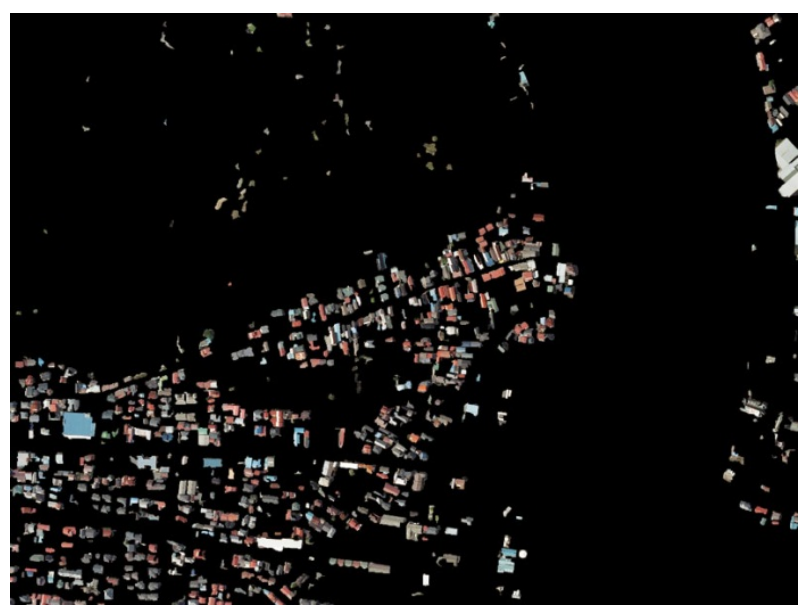

(c)

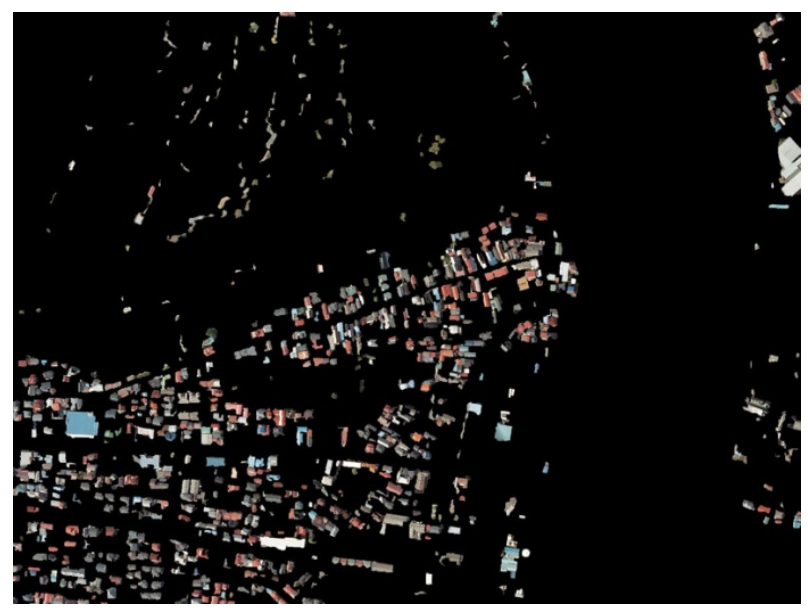

(e)

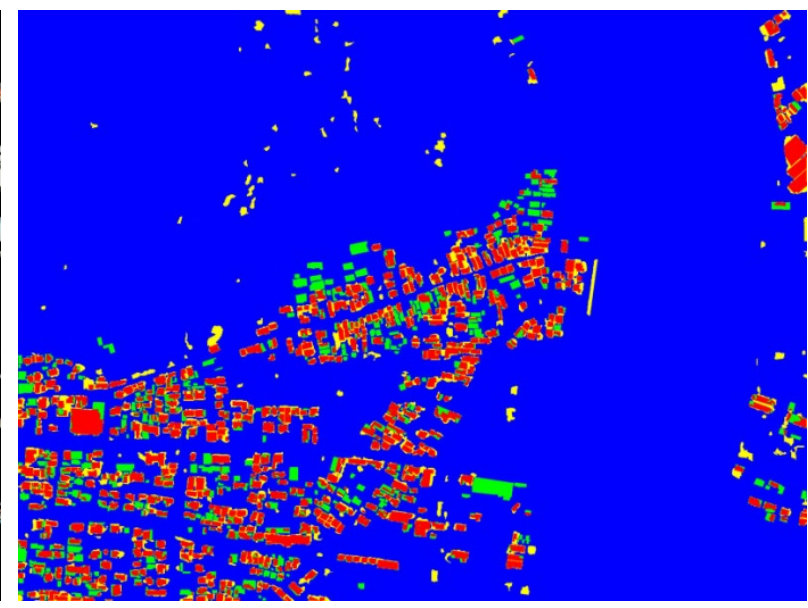

(b)

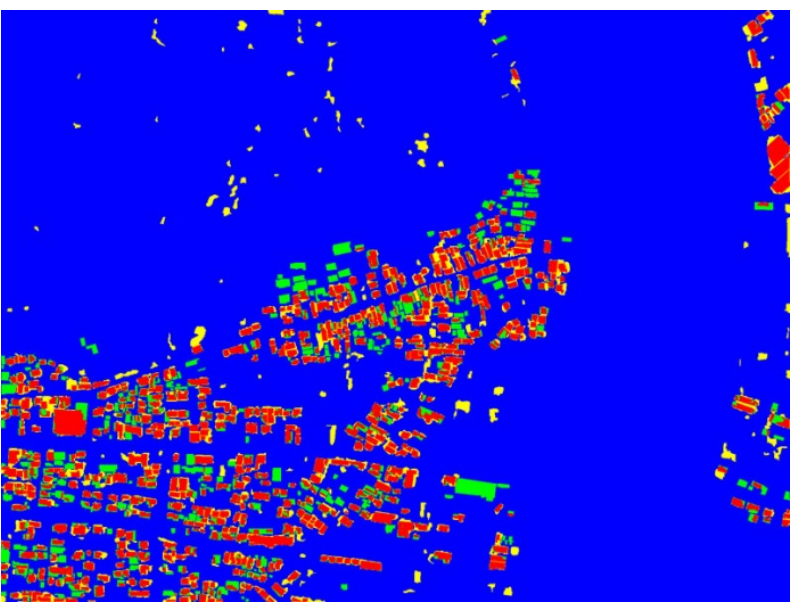

(d)

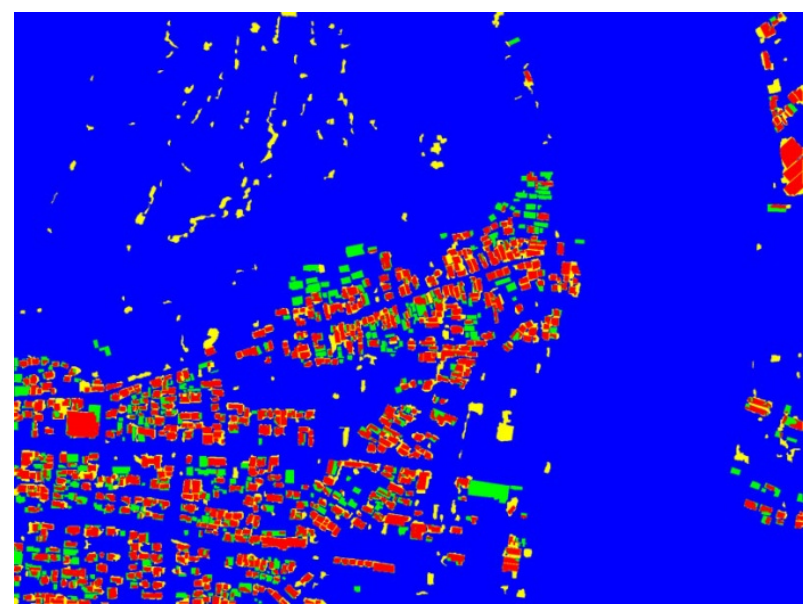

(f)

Figure 11. 

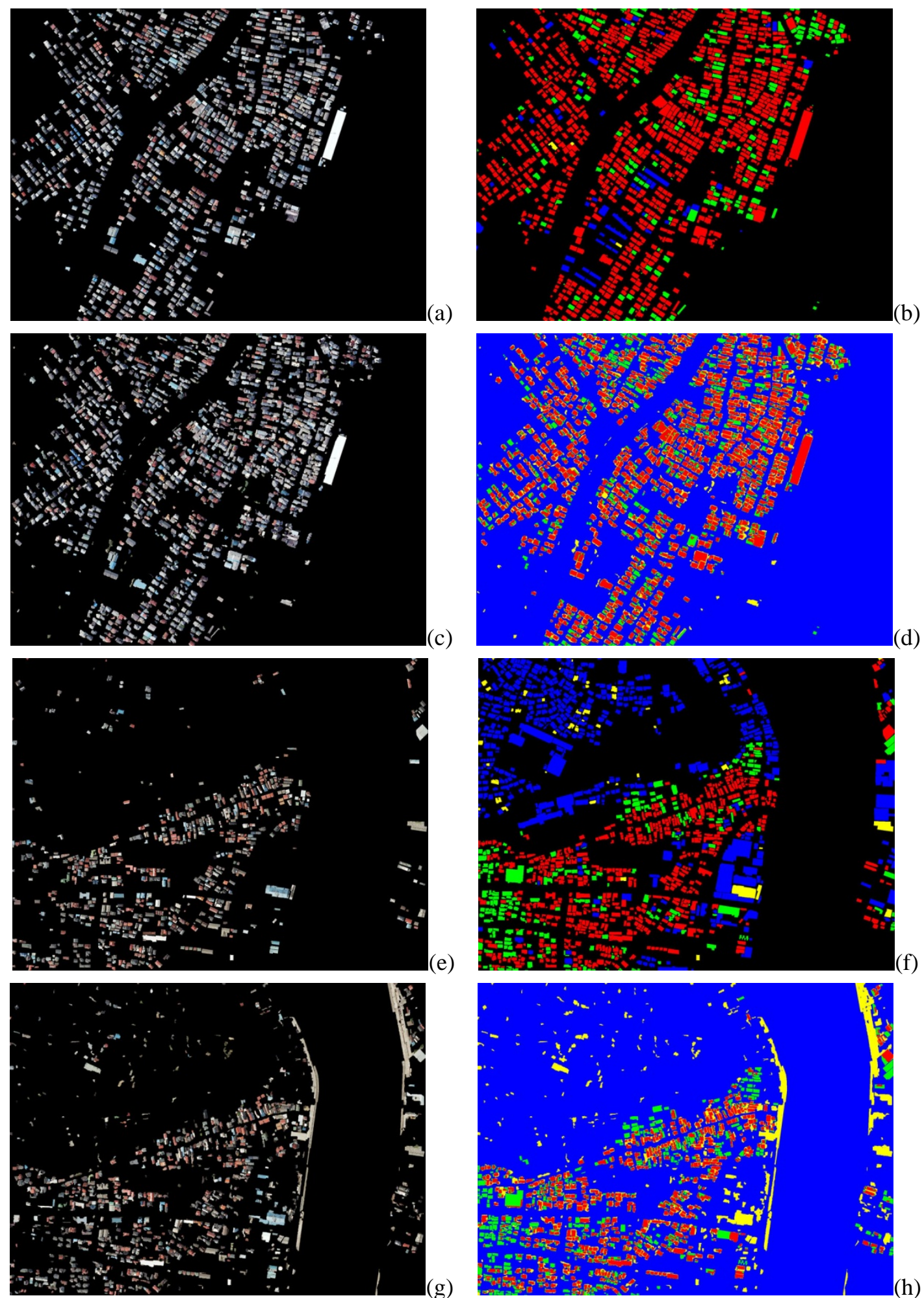

Figure 12. 


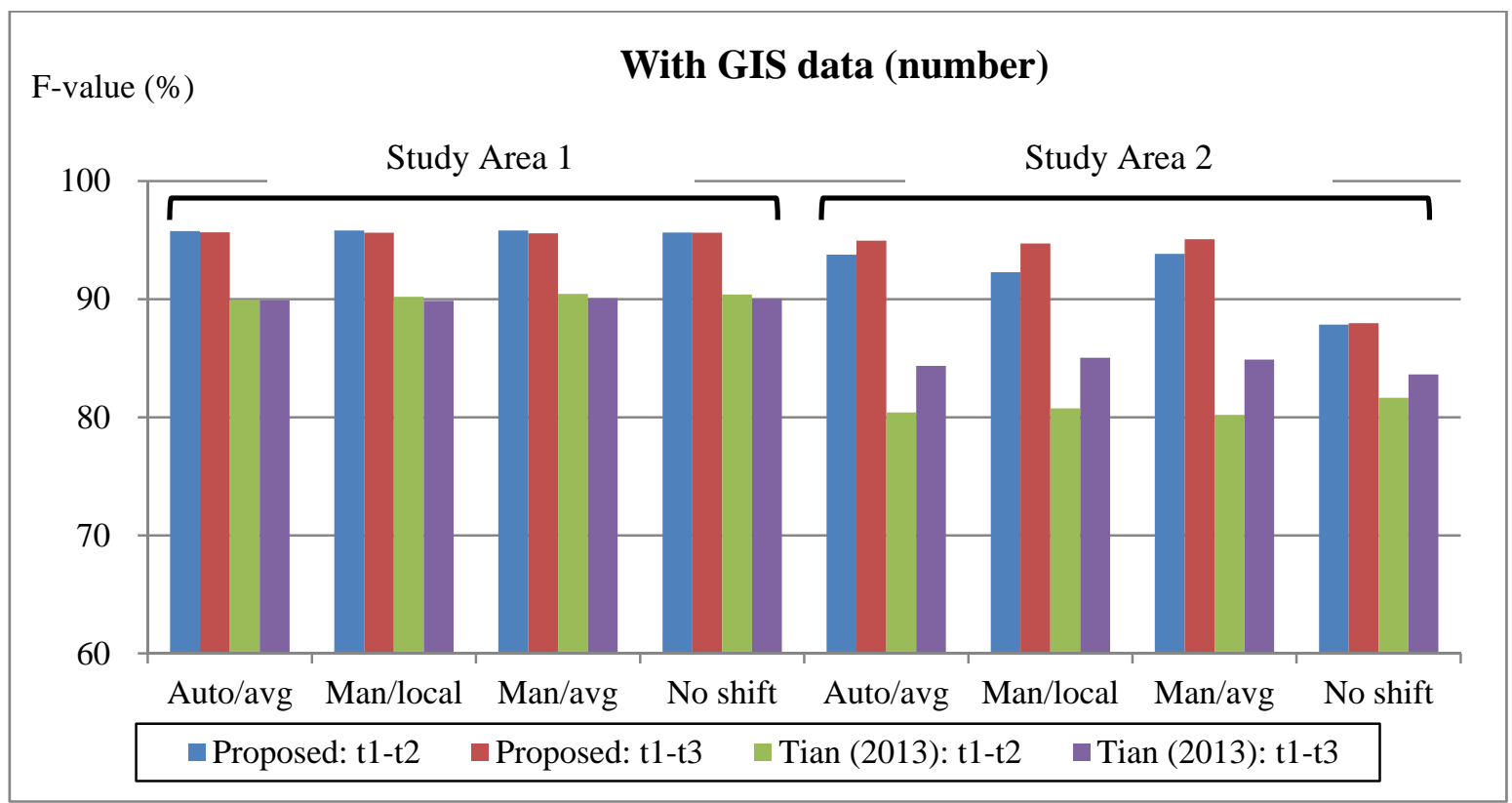

(a)

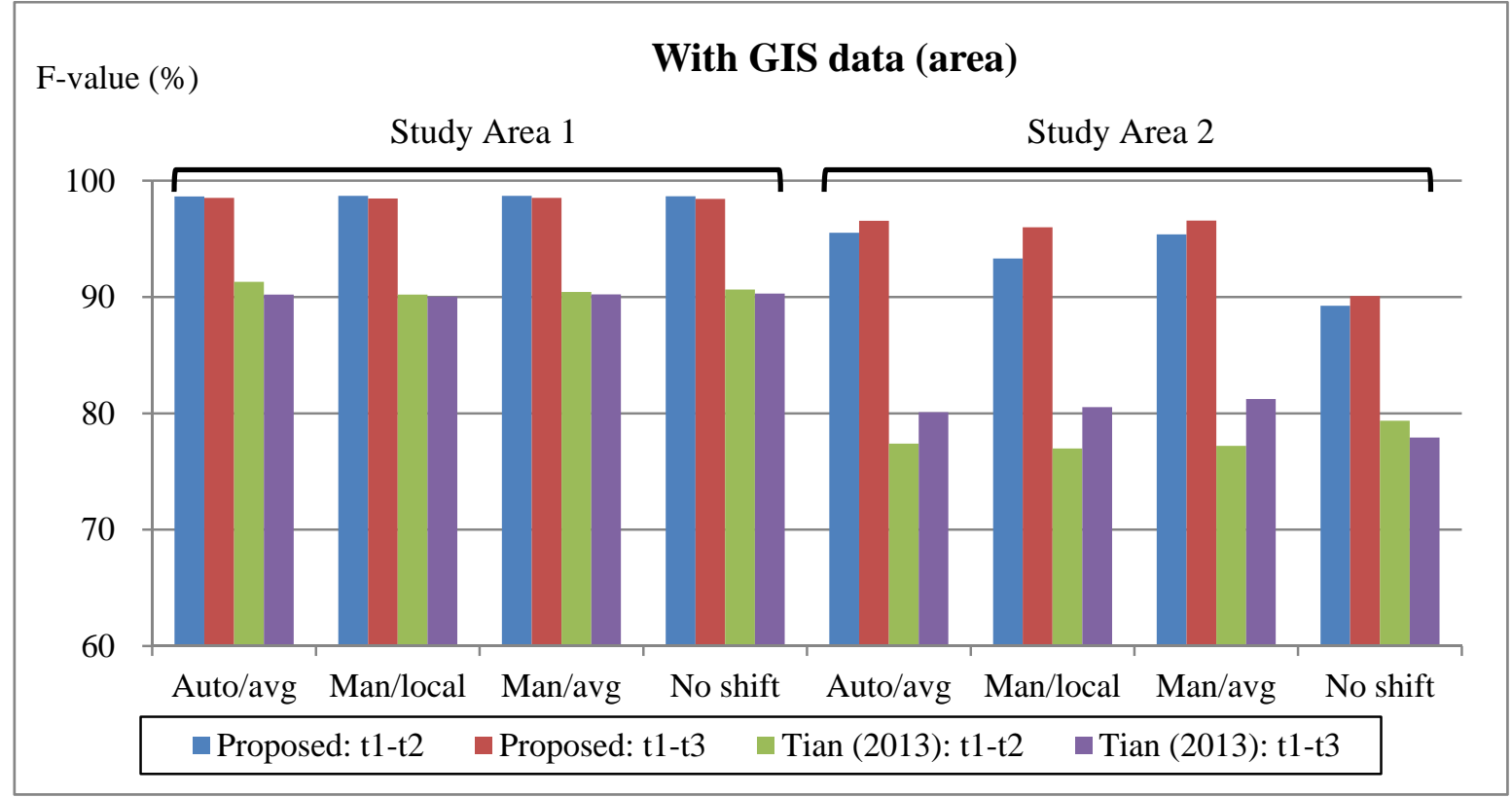

(b)

Figure 13. 


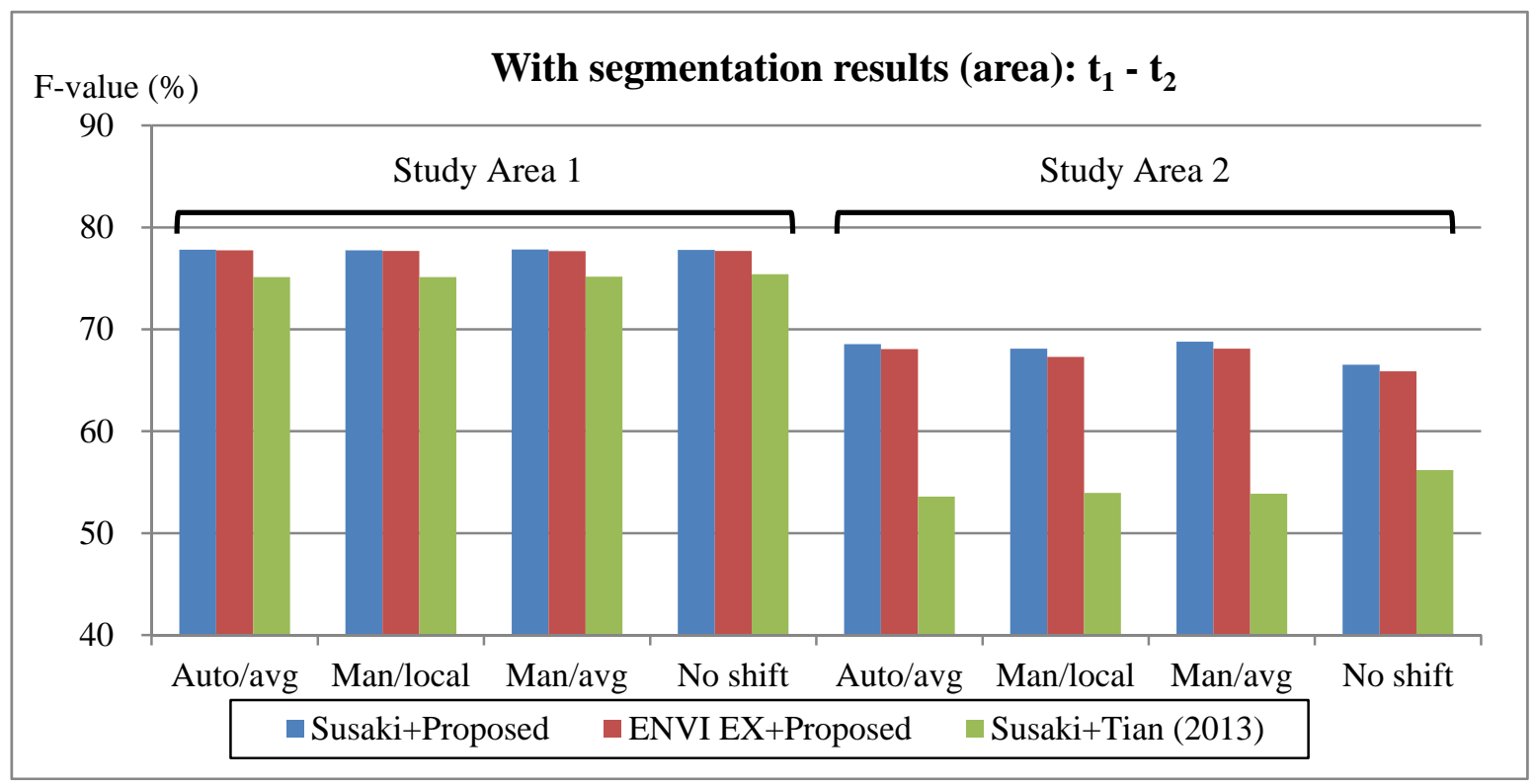

(a)

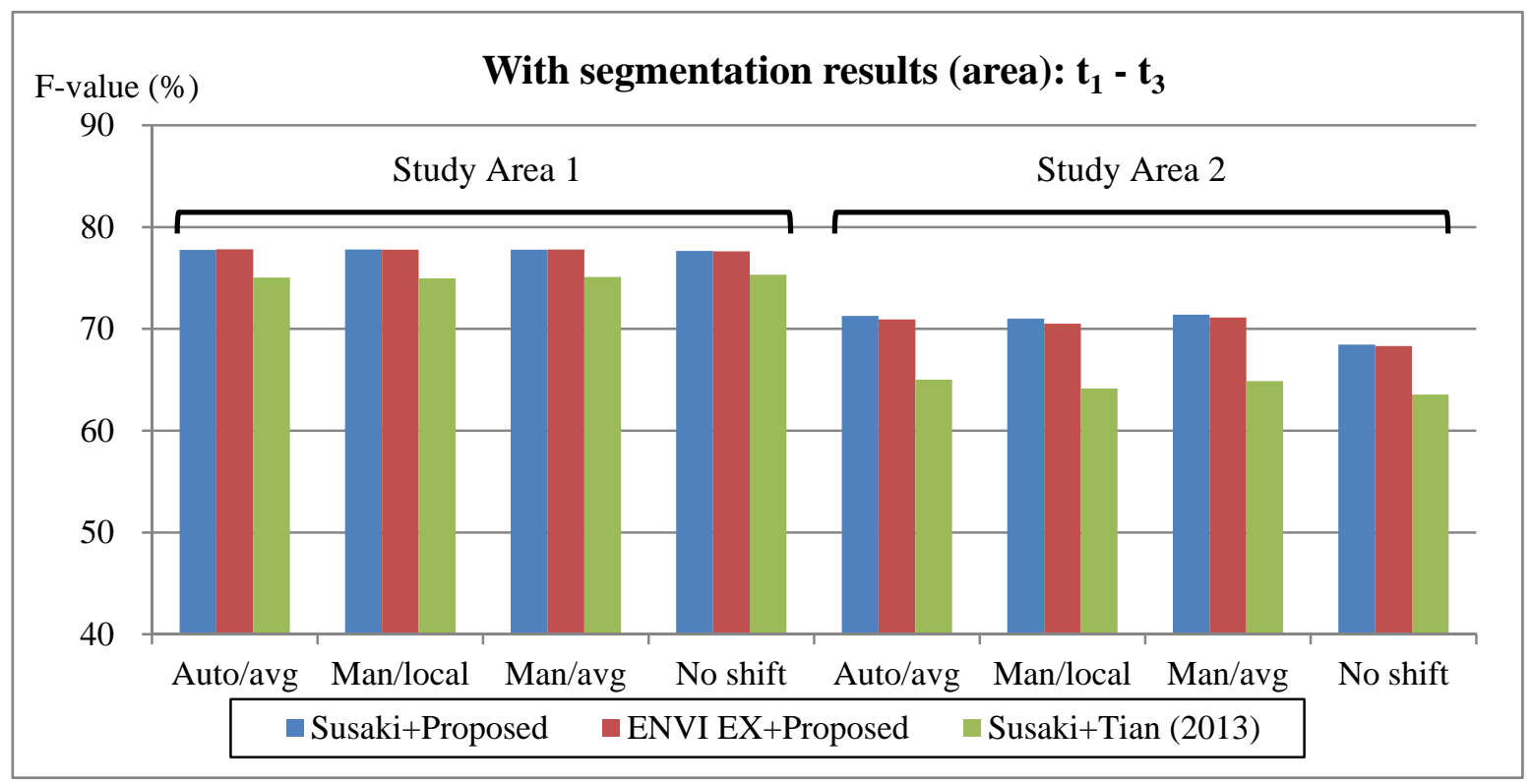

(b)

Figure 14. 
Table 1.

\begin{tabular}{|c|c|c|}
\hline & $\begin{array}{c}\text { May 15, 2009 }\left(t_{1}\right) \text { and } \\
\text { March 12, 2011 }\left(t_{2}\right)\end{array}$ & $\begin{array}{c}\text { May 15, 2009 }\left(t_{1}\right) \text { and } \\
\text { May 26, 2011 }\left(t_{3}\right)\end{array}$ \\
\hline Automatic detection & $(1.35 \mathrm{~m}, 0.35 \mathrm{~m})$ & $(2.55 \mathrm{~m},-1.90 \mathrm{~m})$ \\
\hline Manual detection & $(0.60 \mathrm{~m},-0.27 \mathrm{~m})$ & $(1.10 \mathrm{~m},-1.97 \mathrm{~m})$ \\
\hline
\end{tabular}

Table 2.

\begin{tabular}{|c|c|c|}
\hline & $\begin{array}{c}\text { May 18, 2009 }\left(t_{1}\right) \text { and } \\
\text { March 12, 2011 }\left(t_{2}\right)\end{array}$ & $\begin{array}{c}\text { May 15, 2009 }\left(t_{1}\right) \text { and } \\
\text { May 18, 2011 }\left(t_{3}\right)\end{array}$ \\
\hline Automatic detection & $(3.65 \mathrm{~m},-1.02 \mathrm{~m})$ & $(5.91 \mathrm{~m},-1.45 \mathrm{~m})$ \\
\hline Manual detection & $(2.57 \mathrm{~m},-1.17 \mathrm{~m})$ & $(4.87 \mathrm{~m},-1.27 \mathrm{~m})$ \\
\hline
\end{tabular}

